\title{
Neutron Brillouin scattering and $a b$ initio simulation study of the collective dynamics of liquid silver
}

\author{
Eleonora Guarini $\odot,{ }^{1, *}$ Alessio De Francesco $\odot,{ }^{2}$ Ubaldo Bafile $\odot,{ }^{3}$ Alessio Laloni $\odot,{ }^{4}$ Beatriz G. del Rio $\odot,{ }^{5}$

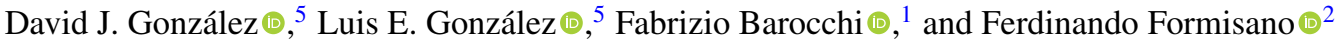 \\ ${ }^{1}$ Dipartimento di Fisica e Astronomia, Università degli Studi di Firenze, via G. Sansone 1, I-50019 Sesto Fiorentino, Italy \\ ${ }^{2}$ Consiglio Nazionale delle Ricerche, Istituto Officina dei Materiali c/o OGG, and Institut Laue Langevin, \\ 71 avenue des Martyrs, CS 20156, F-38042, Grenoble, France \\ ${ }^{3}$ Consiglio Nazionale delle Ricerche, Istituto di Fisica Applicata "Nello Carrara", via Madonna del Piano 10, I-50019 Sesto Fiorentino, Italy \\ ${ }^{4}$ Consiglio Nazionale delle Ricerche, Istituto Officina dei Materiali c/o OGG, and European Spallation \\ Source ERIC, P.O. Box 176, SE-221 Lund, Sweden \\ ${ }^{5}$ Departamento de Fisica Teorica, Atomica y Optica, Universidad de Valladolid, Facultad de Ciencias, \\ Paseo Belen 7, E-47011 Valladolid, Spain
}

(Received 27 May 2020; revised 15 July 2020; accepted 11 August 2020; published 27 August 2020)

\begin{abstract}
We present a thorough investigation of the collective dynamics of liquid Ag combining neutron Brillouin scattering and $a b$ initio molecular dynamics (AIMD) determinations of the dynamic structure factor $S(Q, \omega)$. The main scope of this work is not only to provide experimental results for some important dynamical properties of this liquid metal in the wave-vector range $4<Q<16 \mathrm{~nm}^{-1}$, but also to inquire about the scarce detectability of shear waves apparently characterizing two elements of group IB, differently from other metals. In fact, as in the case of $\mathrm{Au}$, a transverse-like dynamics is not deducible from the experimental $S(Q, \omega)$ of $\mathrm{Ag}$, despite the indisputable quality of the neutron data collected on the BRISP spectrometer at the Institut Laue Langevin in Grenoble. However, the significant agreement between experiment and AIMD calculations allowed for an indepth study of the simulated $S(Q, \omega)$ in a $Q$ range overlapping and extending the experimental one. A multimode analysis, already proven very successful in the description of various dynamical properties of fluid systems, is shown to be extremely effective also to analyze the intermediate scattering function predicted by AIMD at the various $Q$ values, and eventually enables a reliable determination of both longitudinal and transverse branches in the dispersion curve of this liquid. Throughout the paper we highlight the importance of referring to theoretically well-founded models for $S(Q, \omega)$ and of imposing physical constraints in a fit-based analysis: These ensure that the used models obey fundamental properties of the dynamic structure factor.
\end{abstract}

DOI: 10.1103/PhysRevB.102.054210

\section{INTRODUCTION}

The present knowledge about very general dynamic properties of fluids is partly the result of the intense research work specifically devoted to pure liquid metals for decades [1,2]. The simple monatomic nature of these systems is certainly one of the reasons attracting scientists in the field to perform both spectroscopic and simulation studies of this class of liquids (see, e.g., Refs. [3-7] and introductory references therein), which enable to go to the core properties of the translational dynamics without the complications induced by a polyatomic composition of the sample. In many respects, the progresses made up to present times about the physics of the liquid state have seen liquid metals playing a role in experimental studies as important as that of Lennard-Jones (model) fluids in early simulations of whatever many-body disordered system. Therefore, liquid metals represent, from a fundamental point of view, reference samples for experimental investigations, especially when addressing still open issues about the appropriate correlation functions able to reveal the

*Corresponding author: guarini@fi.infn.it main dynamical collective processes in simple liquids, i.e., sound and shear waves. In fact, one longstanding question regards the detectability of transverse-like excitations from what neutron or $\mathrm{x}$-ray $\mathrm{THz}$ spectroscopies provide, i.e., the dynamic structure factor $S(Q, \omega)$. This quantity is related to the longitudinal dynamics [3] and should not, in principle, contain excitations of the transverse type. Nevertheless, although $S(Q, \omega)$ is dominated by longitudinal collective modes, in some systems the presence of a lower-frequency second excitation has been reported [5,8-12] and interpreted as a manifestation of transverse waves propagating in the fluid. This is, in our opinion, a still unsettled issue deserving further investigation. The study of the transverse dynamics in presence of the dominant longitudinal one has been the object of numerical and experimental investigations also in more complex systems as molecular liquids, like, for instance, water [13-15], aqueous mixtures [16], and methanol [17].

At certain densities and length scales, shear modes are unambiguously present also in simple monatomic dense fluids, as confirmed by innumerable simulation results for the density of states of liquids (i.e., the spectrum of the velocity autocorrelation function) or for the transverse current-current correlation, since the late 1970s [18-20] up to more recent 
$[21,22]$ and present times [7,23,24]. Nonetheless, there are contrasting indications about what can effectively be extracted from measurements of $S(Q, \omega)$, if one takes into account that a more or less elaborate data correction involving some approximations, and a sensible choice of the model fit functions are required. The latter point is particularly important if one assumes, as it should be done, that the model spectra must comply with some fundamental properties of $S(Q, \omega)$. In this work we rely on the well-founded theoretical result that assigns to $S(Q, \omega)$ the property of being represented by a sum of generalized Lorentzian lines, as summarized in Sec. IV, with the proviso that such expression should ensure as a minimum requirement that its second frequency moment is a finite quantity. The compliance of fit models with these fundamental properties of $S(Q, \omega)$ does not appear to have always been a concern in the analysis presented in several works on the subject.

Among more or less recent experimental works on liquid metals [4,5,8-12] we find an apparent anomaly about gold, where no transverse-like dynamics was detected via $S(Q, \omega)$ [4], despite its clear evidence in the velocity autocorrelation function (VAF) [24]. In fact, the experimental and simulated Au $S(Q, \omega)$ spectra were satisfactorily reproduced with the generalized hydrodynamic $(\mathrm{GH})$ or viscoelastic (VE) model, respectively. These models, described in Ref. [25], include one inelastic doublet and ensure the finiteness of at least the second spectral moment. Further confirmation that the dynamic structure factor of gold does not carry the fingerprints of shear modes in the experimental $Q$ range was provided by a careful Bayesian analysis of the data [26], revealing that the addition of another inelastic doublet was statistically unjustified, except perhaps at the higher wave vectors of the experiment. At such $Q$ values the posterior distributions of the parameters were still slightly in favor of a one-excitation model, despite providing the interesting indication that a transverse dynamics was not totally excluded, on statistical grounds, at the upper limit of the explored $Q$ range. However, our attempts to fit a double-excitation model to the Au data, while maintaining compliance with the same sum rules of the VE model, either completely failed or provided a worse description even of the $a b$ initio molecular dynamics (AIMD) simulations. We thus found no solid evidence of any detectability of shear waves from the dynamic structure factor of gold, at least within the accuracy of the available experimental and simulation data.

One might, of course, suppose that, differently from the case of other systems like zinc [5], where a tiny feature at low frequency is detectable in the spectra, in metals of the group IB of the periodic table transverse modes are too weak to show up in $S(Q, \omega)$. As just recalled, this appears to be the case of $\mathrm{Au}$ [4]. For $\mathrm{Cu}$, transverse excitations have been reported [12] in a limited $Q$ range from an analysis where they are modeled as Gaussian contributions to the experimentally derived longitudinal current-current spectrum. This can be considered as an empirical method of locating possible excitation frequencies but does not correspond to any known theory of the dynamic structure factor. As we will show in the Appendix, such a kind of analysis can misleadingly induce to explain the lowfrequency shape of the longitudinal current autocorrelation spectrum as due to a real excitation. Therefore, a transverse- like dynamics in liquid $\mathrm{Cu}$ still remains, experimentally, as undetermined as for $\mathrm{Au}$. Understanding whether group IB metals actually represent a sort of exception, as far as the detectability of transverse modes from $S(Q, \omega)$ is concerned, calls of course for an investigation of liquid silver and its dynamical properties.

In this paper we are also interested in addressing a more general problem concerning the interpretation of low- $Q \mathrm{THz}$ frequency spectra in disordered systems, that is, trying to understand the role played by different choices of fit models, and by the imposition or not of physical constraints in the fitting procedures. In the specific case of metallic liquids, apparent differences among various systems might indeed also depend on the data analysis. For instance, results obtained by fitting a two-excitation model to rather noisy data, and with the neglect of basic sum rules [i.e., physical constraints on the moments of $S(Q, \omega)]$ in most cases [8-12], might be not so robust. In this respect, it is important to underline that what appears as an overdamped or close to overdamped harmonic oscillator line shape attributed to a second excitation, and frequently interpreted as transverse in nature, is often, within the typical accuracy of experimental or simulation data, equally or better reproduced, at several $Q$ values, by the second (typically broad) central Lorentzian foreseen by the viscoelastic model [see Eq. (69) of Ref. [25]]. With this we mean that, at least in certain $Q$ ranges, the risk of an overinterpretation of the real information conveyed by $S(Q, \omega)$ cannot be excluded.

To reach more insight about the dynamical properties of liquid metals and the microscopic mechanisms that $S(Q, \omega)$ is able to reveal without ambiguities, we addressed the case of liquid Ag, both experimentally and by ab initio simulations.

AIMD calculations [7] based on density functional theory [27] have provided results for the static structure of liquid silver in remarkable agreement with experimental x-ray [28,29] and neutron [30] diffraction data. By contrast, the dynamic behavior of this liquid metal still lacks an experimental investigation, despite the importance of its alloys in applications [31-33], which by themselves ask at least for a basic knowledge of the main properties of the individual components.

An estimate of the transverse mode frequencies in liquid silver was derived from the analysis of the AIMD transverse current autocorrelation function [7], which undoubtedly reveals the presence of shear waves, as expected given the high density conditions of the fluid. Here our concern is to present experimental results for $S(Q, \omega)$, and to evaluate the ability of AIMD calculations to properly describe also the dynamics of liquid Ag, as already satisfactorily verified for $\mathrm{Au}$ and other liquid metals at ambient pressure, such as Ti [34], Fe [35], $\mathrm{Ni}$, [36], or Zn [37], to mention only some recent studies. Recent AIMD studies have also addressed the case of liquid metals at high pressures, where new dynamic features may appear [38-40], but for which experimental data are mostly still missing.

As mentioned, among our aims is trying to understand what information $S(Q, \omega)$ actually brings when some rigor is applied in the spectral analysis of both experimental and simulation data, and which models are appropriate for silver with varying $Q$, looking for analogies with or differences from the case of gold and other metals. We will show, as in the case 
of other important functions for the dynamics of the liquid state, that a multiexponential analysis [41-43] of the intermediate scattering function $F(Q, t)$ [or, equivalently, a multiLorentzian analysis of the dynamic structure factor $S(Q, \omega)$ ] is once again extremely accurate, ensuring the fulfillment of the first few sum rules along with excellent descriptions of the addressed function, and leading to a clear characterization of the main dynamical properties of the system. Thus the multimode representation is demonstrated here to account very well not only for single-particle (self-) quantities like the VAF, $Z(t),[23,44-46]$, or the self-intermediate scattering function, $F_{\text {self }}(Q, t)$ [24], but also for a collective function as $F(Q, t)$. Of course, the same holds for their respective spectra, $Z(\omega), S_{\text {self }}(Q, \omega)$, and $S(Q, \omega)$. For the sake of clarity and understanding of what follows, it is useful to recall that the mentioned GH and VE models, both corresponding to a (physically constrained) finite sum of Lorentzians, comply with realistic applications of the general multimode representation [41-43]. Indeed, most used models of the second-order memory function $[3,25]$ can be shown to lead to a spectrum given by a sum of an appropriate number of Lorentzian lines [25], although this universal property of the line shape has long remained unnoticed. Going beyond this recognition, our investigation of liquid $\mathrm{Ag}$ will also provide further evidence that, among a variety of such possibilities, the choice of the model to be fitted without unnecessary overparametrizations is becoming an urgent issue in the interpretation of the microscopic physics of fluids [2].

Summarizing, in this work we will show that different approaches currently in use in studies of the microscopic dynamics of liquids, either experimentally [i.e., by spectroscopic measurements of $S(Q, \omega)$ ], or via simulation methods [i.e., accessing $S(Q, \omega)$, the VAF, and the transverse current autocorrelation function] may convey a somehow not unique interpretation of the underlying physics. This difficulty can be overtaken by (1) profitably combining the advantages of both experimental and numerical techniques, (2) performing a data analysis compliant with basic properties of the dynamic structure factor and, in general, of time correlation functions and their spectra, and (3) exploiting, when possible, the aid offered by statistical inference methods, like the Bayesian one [26,47-51].

\section{EXPERIMENT AND ANALYSIS OF THE NEUTRON DATA}

Silver is a relatively strong neutron absorber (absorption cross section [52] $\sigma_{\mathrm{a}}=35.2 \mathrm{~b}$ at $1 \AA$ neutron wavelength), a rather weak scatterer (scattering cross section [52] $\sigma_{\mathrm{s}}=$ $4.99 \mathrm{~b}$ ), and has a high melting temperature $(1235 \mathrm{~K})$, like gold [4]. Our previous experience with Au could thus be fully exploited also for Ag. In particular, the difficulties related to the high melting temperature were again satisfactorily overtaken by choosing the same material, molybdenum (melting temperature $2890 \mathrm{~K}$ [53]), for the realization of the sample container. Moreover, the case of gold constitutes a proof that a high absorption is not an insuperable obstacle for good determinations of $S(Q, \omega)$ by neutron scattering, so the same we expected for silver.
The most interesting $Q$ region in studies of the collective dynamics of liquids typically extends from a few inverse nanometers (where departures from hydrodynamic behavior start to show up also in the dispersion curve of insulating liquids) to values close to the position $Q_{p}$ of the main maximum in the static structure factor (for $\mathrm{Ag}$ [29], $Q_{p}=26.4 \mathrm{~nm}^{-1}$ ), part of which, roughly up to $Q_{p} / 2$, is accessible by means of neutron Brillouin scattering (NBS). NBS investigations of liquids characterized by a high sound velocity like $\mathrm{Ag}$ at melting $\left(c_{\mathrm{s}}=2790 \mathrm{~m} / \mathrm{s}\right.$ [54]) require the use of sufficiently energetic neutrons able to interact with the high-frequency excitation modes of the system. Use of high incident energies in turn implies the need of small angle detection, in order to span the low $Q$ values typical of NBS. The time-of-flight (ToF) spectrometer BRISP at the Institut Laue Langevin (ILL, Grenoble) was designed exactly for this purpose, combining the use of thermal neutrons with a detection area around the direct beam [55-57], and was chosen for the Ag experiment (performed in 2013), as in the case of the previous measurements on $\mathrm{Au}$ and $\mathrm{Zn}[4,5]$.

The sample (25.8 $\mathrm{g}$ of $\mathrm{Ag}$ chunks) was liquefied directly inside the molybdenum slab container (with two walls, each $1.25 \mathrm{~mm}$ thick, orthogonal to the beam direction) placed inside a furnace maintained at $1273 \mathrm{~K}$, i.e., just above the $\mathrm{Ag}$ melting point. The scattering, absorption properties, and the thickness $(3.7 \mathrm{~mm})$ of the $\mathrm{Ag}$ sample correspond to a transmission of $46.5 \%$ at the number density of liquid $\mathrm{Ag}(n=$ $52.1 \mathrm{~nm}^{-3}$ [54]) and at the chosen incident energy discussed below. A boron nitride mask at the entrance wall of the cell was used to reduce the scattering from the container and to properly adjust the area $\left(25 \times 26 \mathrm{~mm}^{2}\right)$ illuminated by the beam.

In order to minimize the effects of absorption and reach the excitation frequencies of liquid $\mathrm{Ag}$, the highest incident energy available on BRISP $\left(E_{0}=83.8 \mathrm{meV}, \lambda_{0}=0.988 \AA\right)$ was selected by exploiting the (004) reflection of the pyroliticgraphite monochromator of the instrument. The detector was placed at $4 \mathrm{~m}$ from the sample position, i.e., at a distance such as to cover scattering angles $\theta$ between $1^{\circ}$ and $15^{\circ}$ and to give a maximum $Q$ value of the order of $Q_{p} / 2$, at the chosen incident wavelength. The choice regarding $E_{0}$ has the obvious drawback that the energy resolution is not very high. However, the same resolution did not prevent, for instance, to observe both longitudinal and transverse modes in liquid zinc [5], and it appears to be sufficient.

As customary in neutron scattering, measurements on a vanadium sample are performed for resolution and normalization purposes. The resolution in the chosen configuration of BRISP was determined by measuring the signal from a vanadium slab (3 $\mathrm{mm}$ thickness) placed inside the empty cell. After correction of the raw $\mathrm{V}$ data for background (including the signal from the empty cell in the furnace), self-attenuation, and multiple scattering, the resulting vanadium spectra were found to have the expected Gaussian shape, with a full width at half maximum of $3.2 \mathrm{meV}$, negligibly dependent on $Q$. The vanadium integrated intensities were determined to allow for the $\mathrm{Ag}$ data normalization to absolute units, exploiting the knowledge of the scattering law (elastic and incoherent) of this important reference sample in neutron scattering. The use of vanadium for such normalization purposes requires to 
keep the geometry as similar as possible in both the $\mathrm{V}$ and Ag measurements, and explains the apparent complication of putting the vanadium foil inside the cell.

The intensities scattered from all the samples (empty cell, vanadium, and liquid $\mathrm{Ag}$ ) have been collected as a function of $\theta$ and of the neutron time of flight, and then converted, using the standard routines available on BRISP (see the Supplemental Material [58]), to 11 constant- $Q I(Q, \omega)$ spectra covering a wave-vector range between 4 and $16 \mathrm{~nm}^{-1}$. The counting rate per unit frequency recorded in the $\mathrm{Ag}$ measurements, once corrected for background, empty cell signal, and selfattenuation, can be written as

$$
I^{\text {expt }}(Q, \omega)=I^{(1)}(Q, \omega)+I^{(\mathrm{m})}(Q, \omega),
$$

where $I^{(1)}(Q, \omega)$ is the single-scattering intensity related to $S(Q, \omega)$, while $I^{(\mathrm{m})}(Q, \omega)$ is the multiple scattering contribution to the total signal (from the sample alone) that one must get rid of to extract the dynamic structure factor from neutron data. Both contributions to the intensity are affected by the instrumental resolution. In our previous paper on gold [4] we commented on the importance of multiple scattering in these kinds of experiments on liquids, since this component deeply affects the tails of the measured spectra and is practically the only surviving contribution at high frequency. We also showed that multiple scattering is not at all negligible in metals, and that a careful correction for this undesired effect is crucial and mandatory. Unfortunately, multiple scattering cannot be measured, so, at least the dominant double scattering intensity from the sample must be calculated as accurately as possible. As in our previous works, we exploited a very efficient algorithm based on multidimensional Monte Carlo integration of appropriate functions [61], that allowed one to calculate the double scattering from the sample, including non-negligible sample-cell cross contributions. We thus derived directly what can be identified with the first term of the right-hand side of Eq. (1).

The single scattering intensity is governed by the neutronscattering law of the sample, i.e., by the neutron double differential cross section $d^{2} \sigma /(d \Omega d \omega)$ of silver. This quantity satisfies the detailed balance condition, and is therefore asymmetric. The general expression of the neutron double differential cross section is

$$
\frac{d^{2} \sigma}{d \Omega d \omega}(Q, \omega)=\frac{k_{1}}{k_{0}}\left[b_{\mathrm{coh}}^{2} S(Q, \omega)+b_{\text {inc }}^{2} S_{\text {self }}(Q, \omega)\right],
$$

where $k_{1}$ and $k_{0}$ are the scattered and incident neutron wave vectors, the $b$ are the coherent and incoherent neutronscattering lengths of the $\mathrm{Ag}$ atomic species, and the neutron combination of the total and self-dynamic structure factors is explicated. So the experimental $I^{(1)}(Q, \omega)$ corresponds to

$$
I^{(1)}(Q, \omega)=C \frac{k_{1}}{k_{0}}\left[b_{\mathrm{coh}}^{2} S(Q, \omega)+b_{\mathrm{inc}}^{2} S_{\text {self }}(Q, \omega)\right] \otimes G(\omega),
$$

where $C$ is the instrumental normalization factor relating the experimental intensity in arbitrary units to the double differential cross section in absolute ones, and we indicated explicitly the convolution with the Gaussian resolution function $G(\omega)$, in order to keep in mind that in the subsequent analysis $I^{(1)}(Q, \omega)$ represents a quantity affected by resolution broadening.

In order to derive $S(Q, \omega)$ from Eq. (3) one finally needs a reliable determination of $S_{\text {self }}(Q, \omega)$. The neutron coherent and incoherent cross section values of $\mathrm{Ag}\left(\sigma_{\mathrm{coh}}=4 \pi b_{\mathrm{coh}}^{2}=\right.$ $4.41 \mathrm{~b}$ and $\sigma_{\text {inc }}=4 \pi b_{\text {inc }}^{2}=0.58 \mathrm{~b}$ ) might misleadingly suggest a relatively small incoherent signal from this system. On the contrary, incoherent scattering largely influences the neutron intensities whenever the coherent component, whose integral is proportional to $S(Q)$, is probed at the rather small $Q$ values of NBS, where the static structure factor remains close to $S(0)$ which, for dense liquids, is typically rather small ( 0.016 for $\mathrm{Ag}$ at the melting point), due to the very low compressibility of the liquid. Thus, $S_{\text {self }}(Q, \omega)$, whose frequency integral is 1 independently of $Q$, often gives rise to the dominant signal in the experimental NBS spectra of nominally "coherent" samples.

To deal with this last step of the analysis, we performed, $Q$ by $Q$, a global fit to the experimental $I^{(1)}(Q, \omega)$ able to provide us with a simultaneous estimate both of the normalization constant $C$ (which turned out to be in agreement with the estimate obtained from the vanadium measurements within $15 \%-20 \%$, as typically happens because the vanadium determination of $C$ is affected by the uncertainties on the effective number of illuminated atoms) and of the parameters of some possible modeling of $S(Q, \omega)$, e.g., a classical GH model as a first trial, taking of course asymmetrization and resolution into account. Concerning the self-term, we instead imposed, in the fit algorithm, the shape of $S_{\text {self }}$ to be the experimental-like equivalent of the one obtained from our AIMD simulations. This choice is suggested by the fact that our experimental $Q$ values are not low enough to assume the Lorentzian hydrodynamic line shape predicted by Fick's law [3]. This is not at all a critical point since, after broadening, $S_{\text {self }}(Q, \omega) \otimes G(\omega)$ is substantially the resolution function $G(\omega)$ itself of the instrument. In fact, it is well known that when hydrodynamic behavior is lost, which is certainly the case at our highest experimental $Q$, the increase of the width with $Q$ is weaker than the quadratic growth valid in the hydrodynamic regime [see, e.g., Ref. [2] at Chap. 7, Fig. 7.10, and Ref. [64], Fig. 9(b)]. By using the diffusion coefficient $D$ available in the literature for silver at melting $\left(D=2.55 \times 10^{-9} \mathrm{~m}^{2} / \mathrm{s}\right.$, according to Ref. [65]), we can then take the full width at half maximum predicted by Fick's law, namely, $2 D Q^{2}$, as an upper bound for the width of the actual $S_{\text {self }}$. In this way we obtain a value of $0.86 \mathrm{meV}$ at our highest $Q$, which is seen to remain well below the experimental resolution full width $(3.2 \mathrm{meV})$. So the modeling of $S_{\text {self }}$ is dominated by resolution. However, by using the simulated (broadened) line shape in place of the normalized resolution function it is possible to catch the slight $Q$ dependence of $S_{\text {self }}(Q, \omega) \otimes G(\omega)$, while resolution is $Q$ independent.

In a first attempt to fit the right-hand side of Eq. (3), the $\mathrm{GH}$ (symmetric) model has been used for $S(Q, \omega)$. This can be written as [25]

$$
\begin{aligned}
S_{\mathrm{GH}}(Q, \omega)= & \frac{S(Q)}{\pi}\left[a_{0} \frac{z_{0}}{z_{0}^{2}+\omega^{2}}+a_{\mathrm{s}} \frac{z_{\mathrm{s}}+b_{\mathrm{s}}\left(\omega+\omega_{\mathrm{s}}\right)}{z_{\mathrm{s}}^{2}+\left(\omega+\omega_{\mathrm{s}}\right)^{2}}\right. \\
& \left.+a_{\mathrm{s}} \frac{z_{\mathrm{s}}-b_{\mathrm{s}}\left(\omega-\omega_{\mathrm{s}}\right)}{z_{\mathrm{s}}^{2}+\left(\omega-\omega_{\mathrm{s}}\right)^{2}}\right],
\end{aligned}
$$




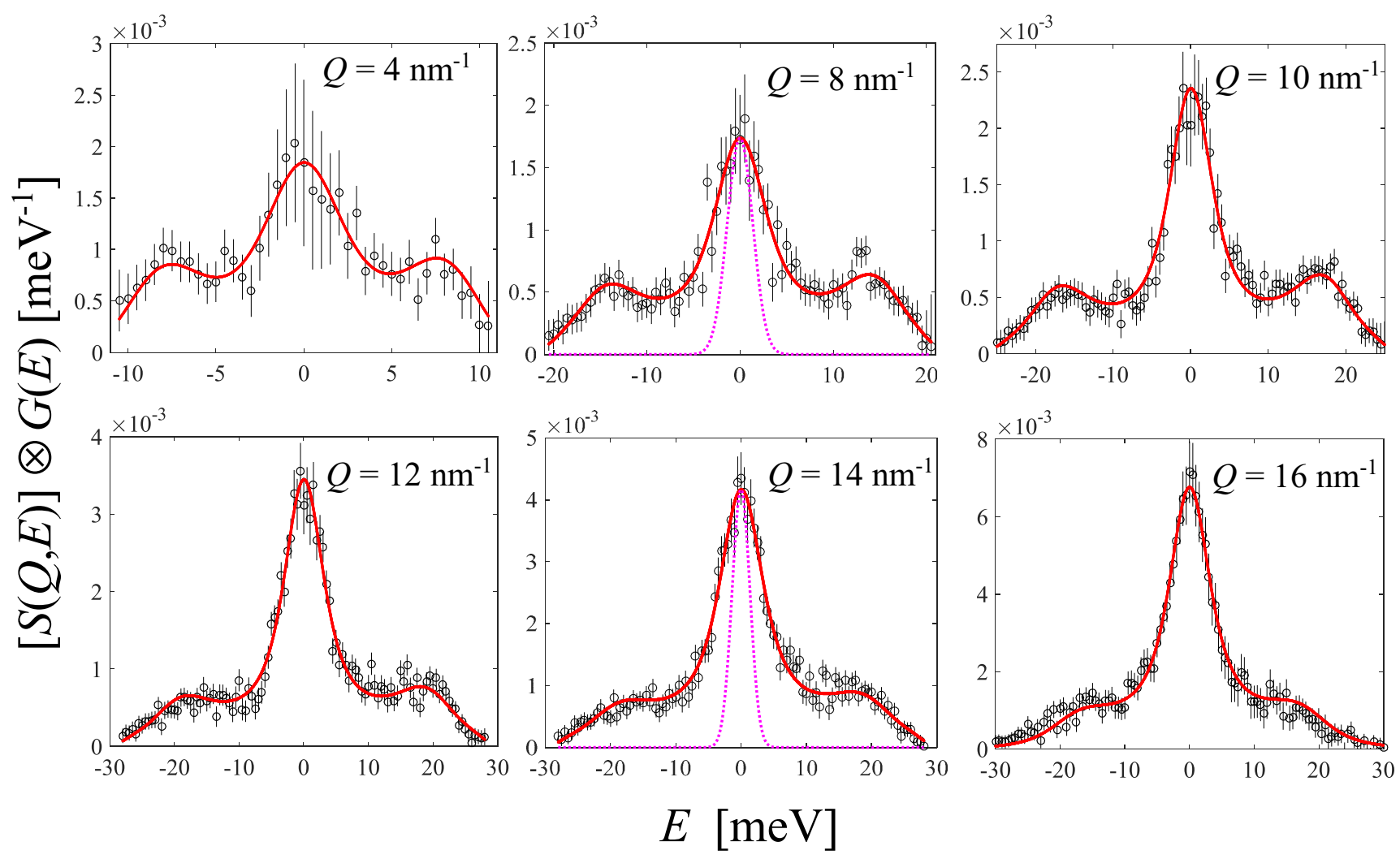

FIG. 1. Dynamic structure factor of silver at six selected $Q$ values of the neutron experiment. The experimental data (black circles with error bars) are affected by resolution broadening. The corresponding fit by means of the GH model (red solid line) has been carried out taking detailed-balance asymmetry and resolution into account. A comparison with the Gaussian instrumental resolution function (scaled to the central peak height, pink dotted curve) is provided at two example $Q$ values.

where the $Q$ dependence of the fit parameters $a_{0}, z_{0}, z_{\mathrm{s}}$, and $\omega_{\mathrm{s}}$ was omitted to shorten the notation. In Eq. (4), $a_{0}$ and $z_{0}$ denote, respectively, the amplitude and half-width of the central Lorentzian line representing, in the $Q \rightarrow 0$ limit, the thermal nonpropagating mode, while $z_{\mathrm{s}}$ is the damping and $\omega_{\mathrm{s}}$ the frequency of the sound modes. The other parameter $b_{\mathrm{s}}$ in Eq. (4) can be calculated from the fitted ones [25]. Given the very good agreement of the AIMD $S(Q)$ with diffraction data [7], we took the simulation result for this quantity in Eq. (4), minimizing the number of free parameters of the final fit function, which reads

$$
\begin{aligned}
I^{\mathrm{fit}}(Q, \omega)= & C \frac{k_{1}}{k_{0}}\left\{B ( \omega ) \left[b_{\mathrm{coh}}^{2} S_{\mathrm{GH}}(Q, \omega)\right.\right. \\
& \left.\left.+b_{\mathrm{inc}}^{2} S_{\text {self,AIMD }}(Q, \omega)\right]\right\} \otimes G(\omega),
\end{aligned}
$$

where $B(\omega)$ is the Bose asymmetrization factor given by

$$
B(\omega)=\frac{\beta \hbar \omega}{1-e^{-\beta \hbar \omega}},
$$

with $\beta=\left(k_{\mathrm{B}} T\right)^{-1}, k_{\mathrm{B}}$ the Boltzmann constant, and $\hbar$ the reduced Planck constant. In principle, asymmetrization should precede resolution broadening, although doing the reverse does not lead to significant errors.

In Fig. 1 we report the so-derived dynamic structure factor of $\mathrm{Ag}$, normalized to absolute units, at some $Q$ values of the measurements. In Fig. 1 we also plot the GH fit results, which give a very good account of the experimental line shape at all wave vectors. We note in passing that, as in other works, we chose to show the quantities strictly related to the experiment as a function of the exchanged energy $E=\hbar \omega$ in units of meV just to facilitate the understanding of the experimental ranges and widths by experimentalists. Differently, in the remainder of this paper, we will use as spectral variable the angular frequency $\omega$ in units of $\mathrm{rad} \mathrm{ps}^{-1}$ for an easier comparison with other published data.

It is an established fact (see, e.g., Refs. [64,66] that a GH modeling is not working properly outside the long-wavelength region, where viscoelasticity comes in, and the central peak in the spectrum needs to described by the superposition of two nonpropagating modes, as done in the VE model. However, our attempts to fit to the experimental data more complicated models (with growing number of free parameters) like the VE one failed because, within the experimental errors and with the present resolution, the additional parameters turned out to be nonsignificant, i.e., undetermined, with errors on the parameters equal to or larger than the parameters themselves. This descends from the fact that the experimental signal due to the collective modes is only a small part of the total neutron intensity, owing to the incoherent scattering contribution. Consequently, the finite accuracy and resolution of the total neutron data, along with the presence of the incoherent component, makes it very difficult to parametrize significantly the fine structure of the central part of the spectrum. Therefore, the resulting experimental width of the central component cannot be identified with any genuine relaxation process, but 


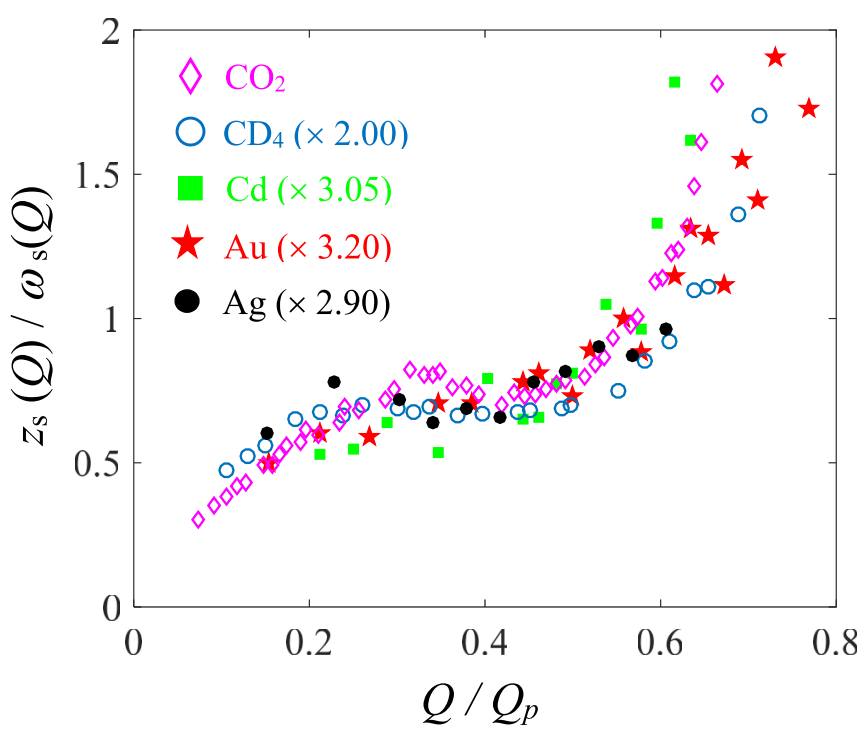

FIG. 2. Ratio of the damping to the frequency of the longitudinal acoustic excitation for insulating and conducting liquids we analyzed in the past [4] with the addition of the Ag results of the present experiment. Data for each system have been scaled to the carbon dioxide values because of the different sound velocities (i.e., multiplied by 3.05 for $\mathrm{Cd}, 3.20$ for $\mathrm{Au}, 2.9$ for $\mathrm{Ag}, 2$ for $\mathrm{CD}_{4}$ ) and plotted as a function of $Q / Q_{p}$. The various symbols in the figure are assigned as specified in the legend. Full and empty symbols are used to help distinguishing the liquid metals from the nonconductive fluids.

only with an unknown combination of damping coefficients. Thus, as in the case of Au, NBS experimental data for the $S(Q, \omega)$ of Ag allow only for a simple $\mathrm{GH}$ description. However, our main concern regards propagating excitations and, as shown for $\mathrm{Au}$, the frequencies determined from the $\mathrm{GH}$ and VE analyses of neutron and simulation data, respectively, were very similar to each other, and a rather small positive dispersion was detected (see Fig. 7 of Ref. [4]). For analogous reasons it was not possible to fit to the experimental spectra models including additional parameters required to account for a second propagating excitation.

As we will show in Sec. IV, the experimental dispersion curve and damping dependence on $Q$ of the sound mode, as obtained by the above GH description of the measured spectra, resemble very much those of gold and of other simple liquids, beyond the differences in the sound speed of the various systems. This is confirmed by Fig. 2 where the present Ag experimental results are in more than satisfactory agreement with the $z_{\mathrm{s}} / \omega_{\mathrm{s}}$ "master" curve we originally reported in Ref. [4]. The plotted curves were obtained by multiplying the $z_{\mathrm{s}} / \omega_{\mathrm{s}}$ values by $2.00,3.05,3.20$, and 2.9 for $\mathrm{CD}_{4}, \mathrm{Cd}, \mathrm{Au}$, and $\mathrm{Ag}$, respectively, keeping $\mathrm{CO}_{2}$ as the reference system. Thus, $z_{\mathrm{s}} / \omega_{\mathrm{s}}$ in simple nonmetallic liquids is larger than in liquid metals, but simple scaling factors bring the ratios for the various systems to lie on the same curve in the whole $Q$ range. The variation of such scale factors is, as mentioned, mainly related to the spread of the sound velocities among the various fluids.

Again, such results tell us that, at the length and time scales probed by $\mathrm{THz}$ spectroscopy, no striking difference can be found in the dynamics of conducting and insulating liquids, as far as the properties of the acoustic excitations are concerned. Therefore, it appears to be confirmed that these techniques, and the $Q$ ranges they are able to probe, give information only on the ionic dynamics, without visible effects originating from the electronic behavior of such different systems from a macroscopic point of view.

\section{AIMD SIMULATIONS AND COMPARISON WITH NEUTRON DATA}

The availability of the neutron measurements permits now to verify the AIMD predictions for liquid Ag also at a dynamical level. Details of the calculations for this liquid metal were given in Ref. [7]. Here it is useful to recall that the simulated sample contains $150 \mathrm{Ag}$ atoms, with eleven valence electrons each, in a periodically repeated cubic cell of adequate length to match the experimental number density. As a consequence, the minimum wave vector compatible with the periodicity is $4.4 \mathrm{~nm}^{-1}$. An ultrasoft pseudopotential to describe the electron-ion interaction was specifically constructed using the tools included in the Quantum Espresso package [67], which we used to perform the simulations. The local density approximation for exchange and correlation was employed, and nonlinear core corrections were explicitly taken into account. A total of 19000 equilibrium configurations were generated, amounting to $142.5 \mathrm{ps}$ of simulation time, from which we obtained the dynamic properties of interest using their standard microscopic definitions [3].

The comparison between the experimental $S(Q, \omega)$ and the corresponding AIMD calculations, duly broadened to take the experimental resolution into account, is reported in Fig. 3. Agreement between data and calculations is quite satisfactory at all $Q$ values and authorizes deepening the analysis of the dynamical behavior of this liquid metal by performing fits to the simulations in a broader wave-vector range (between 4.4 and $48.5 \mathrm{~nm}^{-1}$ ) than the one probed in the experiment. Therefore, in the following, we will present a more refined analysis of the simulated $S(Q, \omega)$ also outside the experimental range.

Indeed, the GH model fitted to the neutron data is able to capture quite effectively the longitudinal dynamics up to $Q_{p} / 2$, but cannot, by construction, give any information about either a viscoelastic behavior or the presence in the fluid of other propagating excitations. In this respect, it is useful to note that small systematic differences can be observed between the GH description of the neutron data and the AIMD outputs of Fig. 3, although well within the experimental uncertainties. For clarity, we show in Fig. 4 the direct comparison between the GH fit curve displayed in Fig. 3 and the AIMD equivalent at three additional $Q$ values (note that we switched to angular frequency in place of energy). There, it is seen that the shape of the simulated spectra is slightly different from the GH one. While a small underestimate by AIMD of the main central peak is observed only at some $Q$ values, the side peaks are systematically less intense and slightly broader than in the GH case. Consequently, at frequencies approximately in the range between 5 and $18 \mathrm{rad} \mathrm{ps}^{-1}$, the $\operatorname{AIMD} S(Q, \omega)$ is seen to exceed the GH one. We took this as an indication that in order to accurately reproduce the simulated spectra at our $Q$ values the simple triplet line shape should be abandoned, as it happened also for gold. The differences between a GH 


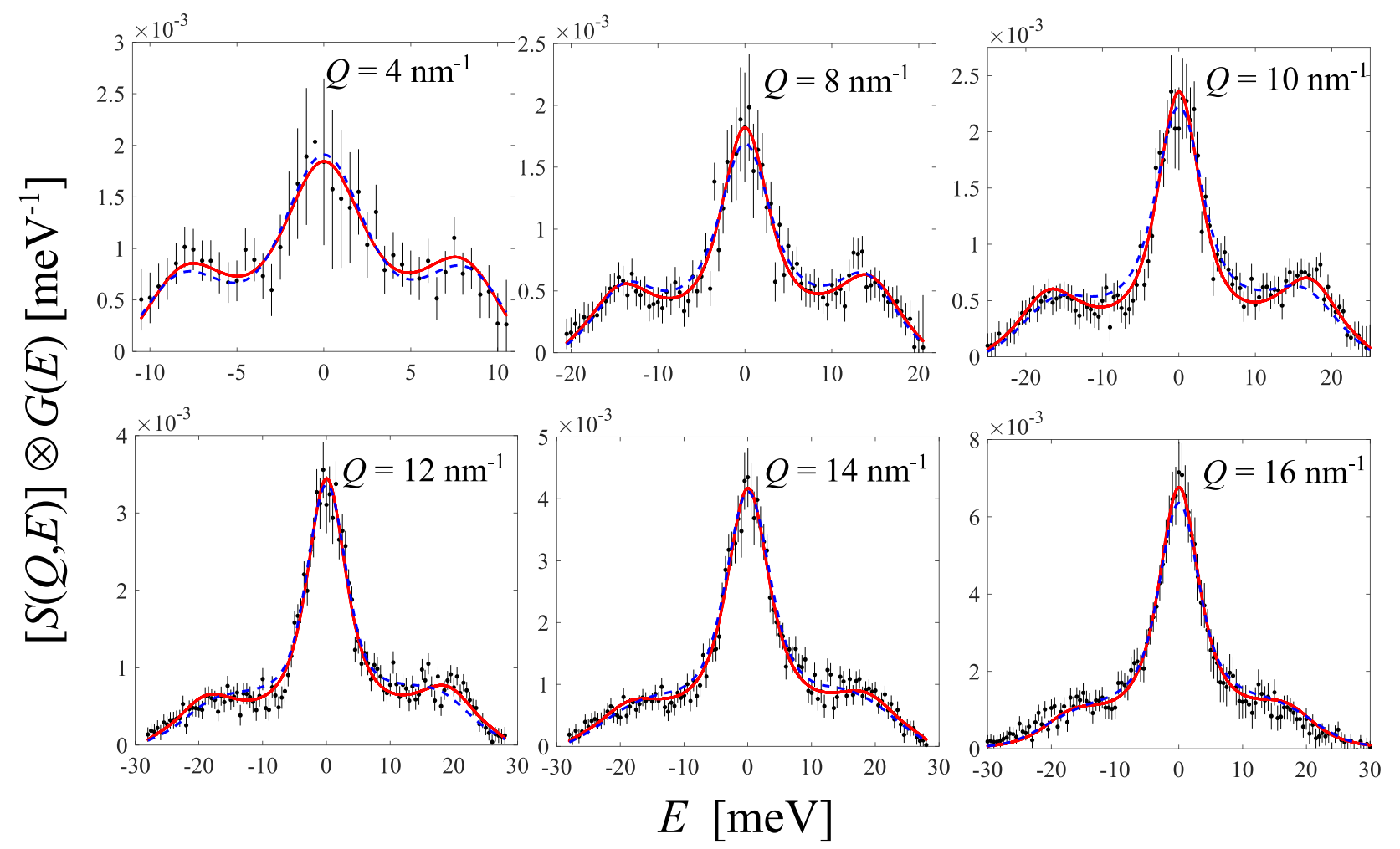

FIG. 3. Same as Fig. 1, with the addition of the AIMD results (blue dashed curve) at the various $Q$ values. The neutron data points (black dots) have been reduced in size in order to increase the visibility of the figures. Data asymmetrization and experimental resolution broadening have been applied to the original AIMD results in order to allow for the comparison.

and a VE fit to the simulated $S(Q, \omega)$ shown in Fig. 6 of Ref. [4] resemble very much those found for Ag. Therefore, in the following we will refer to more complex models, as anticipated in the Introduction.

\section{MULTIMODE ANALYSIS OF THE AIMD SIMULATIONS}

In recent years we have shown the effectiveness of the exponential series representation [41-43] in accounting for the behavior of time correlation functions of classical $[23,24,44]$ and quantum $[45,46]$ fluids. The power of the method lies not only in its providing excellent descriptions of the most relevant functions in liquid state physics, but also in its merit to facilitate the imposition of, at least a few, physical constraints, thus largely increasing the reliability of the results. Here we explore the performance of this multimode decomposition for a collective quantity like the intermediate scattering function $F(Q, t)$ or, equivalently, its spectrum $S(Q, \omega)$.

We recall that $F(Q, t)$ is the autocorrelation of the Fourier components of the microscopic density [68],

$$
F(Q, t)=\frac{1}{N}\left\langle\sum_{\alpha, \beta=1}^{N} e^{-i \mathbf{Q} \cdot \mathbf{R}_{\alpha}(0)} e^{i \mathbf{Q} \cdot \mathbf{R}_{\beta}(t)}\right\rangle,
$$

where $N$ is the total number of atoms, $\mathbf{R}_{\alpha}(0)$ is the position of the $\alpha$ th atom in the (arbitrarily chosen) time origin, and $\mathbf{R}_{\beta}(t)$ is the position of another particle (however note that the case $\alpha=\beta$ is included in the definition) at a subsequent time $t$. The angle brackets denote, as usual, a canonical ensemble average. The isotropy of the fluid actually makes this function depend only on the modulus $Q$ of $\mathbf{Q}$, as indicated in the first member of Eq. (7).

According to the quoted exact theory, the intermediate scattering function has, like any other classical or semiclassical time correlation function, an infinite series representation involving, in general, complex exponentials. For mere convenience, we refer to its normalized values, and we synthetically write it in the form

$$
F_{\mathrm{n}}(Q, t)=F(Q, t) / F(Q, 0)=\sum_{j=1}^{\infty} I_{j} \exp \left(z_{j}|t|\right),
$$

where of course $F(Q, 0)=S(Q)$, and $I_{j}$ and $z_{j}$ are allowed to take complex values.

Equation (8) establishes the exact functionality of the autocorrelation, but not the specific amplitudes $\left(I_{j}\right)$ and dampings/frequencies $\left(z_{j}\right)$ of the "modes" governing the dynamics of the given system. This fact should not be interpreted as a limitation of the method or of the theory: It is worth clarifying that our approach privileges the idea that the data should, so to say, "speak by themselves" and that the dominant physical processes acting in the liquid should be detected from an analysis, as direct as possible, of the relevant correlation functions. The knowledge of the correct functionality of whatever time correlation function thus provides us with a theoretically valid tool for defining plausible, justifiable, and realistic fitting models having the great advantage of being applicable, indifferently, to either measured or simulated data. This knowledge is very valuable and represents a solid basis for trusting the significance of the results. In particular, we 


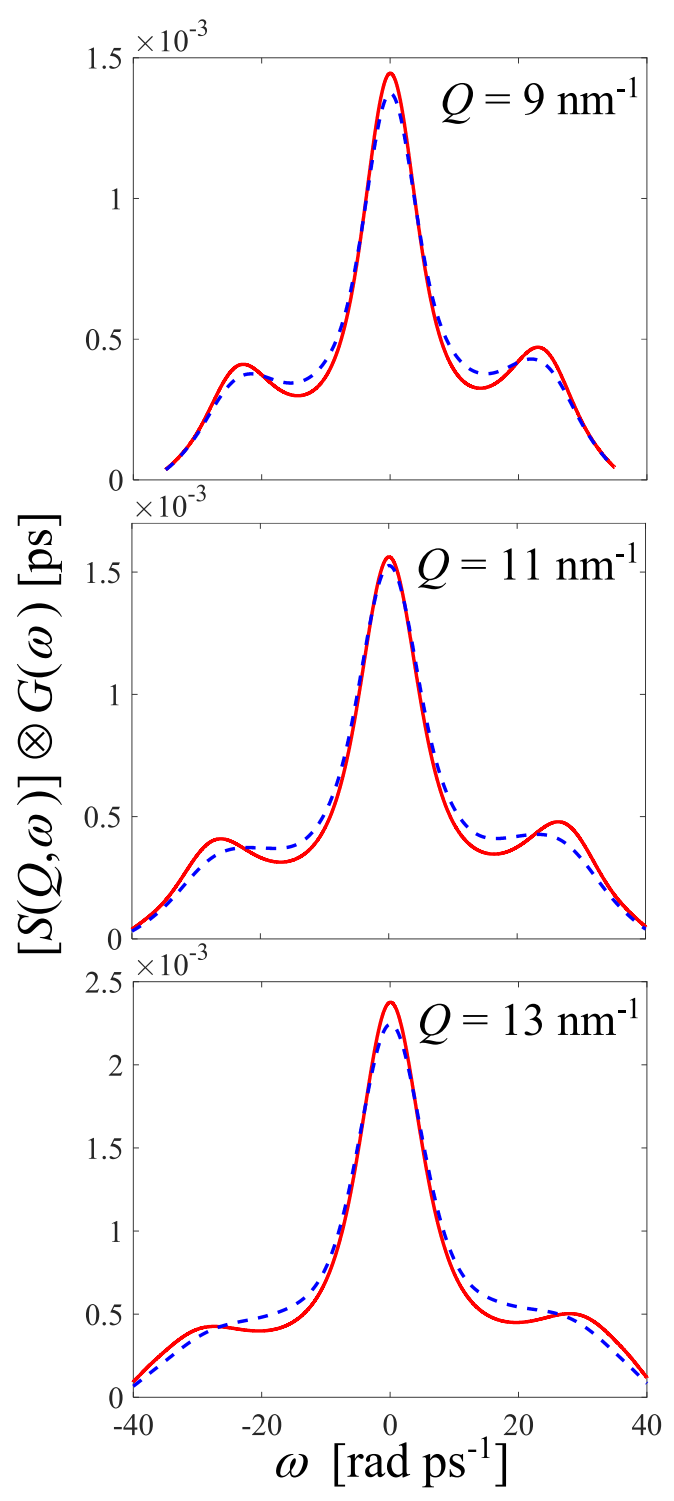

FIG. 4. GH fit to the neutron $S(Q, \omega)$ data (red solid curve) and AIMD calculation (blue dashed curve) at three example $Q$ values.

will show in the following that a reliable characterization of the collective excitations, which is the main goal of our analysis, is achievable through the comparison of various correlation functions.

In $F_{\mathrm{n}}(Q, t)$, as defined by Eq. (8), pure exponential decays are accounted for in the series by what we will refer to as "real modes", i.e., having both $I_{j}$ and $z_{j}$ real, with $z_{j}<0$. Damped oscillatory modes of $F_{\mathrm{n}}(Q, t)$ are represented in the series by what we will designate as "complex pairs", i.e., by $I_{j} \exp \left(z_{j}|t|\right)+I_{j}^{*} \exp \left(z_{j}^{*}|t|\right)$, with both $I_{j}$ and $z_{j}$ complex, and $\operatorname{Re} z_{j}<0$.

Given the representation of Eq. (8), the corresponding frequency spectrum reads

$$
\frac{S(Q, \omega)}{S(Q)}=\sum_{j=1}^{\infty} L_{j}(\omega)=\sum_{j=1}^{\infty} \frac{I_{j}}{\pi}\left[\frac{\left(-z_{j}\right)}{\omega^{2}+z_{j}^{2}}\right],
$$

where $L_{j}(\omega)$ is a "generalized" Lorentzian line. If $I_{j}$ and $z_{j}$ are real, then $L_{j}(\omega)$ is a genuine Lorentzian centered at $\omega=0$, and characterized by a half width at half maximum equal to $-z_{j}$. If $I_{j}$ and $z_{j}$ are complex, then the corresponding mode and its complex conjugate add up to give a pair of distorted Lorentzians centered at the nonzero frequencies $\pm \operatorname{Im} z_{j}$ [see Eq. (4) of Ref. [24] for details]. Finally, it is useful to recall that the normalization of $F_{\mathrm{n}}(Q, t)$ to its initial value leads to the sum rule $\sum_{j=1}^{\infty} I_{j}=1$, and that the existence of its $k$ th order $(k=0,1,2, \ldots)$ time derivatives at $t=0$ leads, in the exponential representation of $F_{\mathrm{n}}(Q, t)$, to an infinite set of sum rules of the form

$$
\sum_{j=1}^{\infty} I_{j} z_{j}^{k}=\left.\frac{d^{k} F_{\mathrm{n}}(Q, t)}{d t^{k}}\right|_{t=0}=0
$$

for odd $k$.

Applications of the mode decomposition method of course require a truncation of the infinite series of Eq. (8). As we showed in our previous papers $[23,24,44,46]$, the remarkable thing is that only a small number of real and complex terms is typically required to obtain excellent descriptions of the addressed correlation, meaning that the main dynamical features originate from a few microscopic processes. Therefore, like in other cases, the analysis of the time dependence of $F(Q, t)$ is similarly performed here by fitting the sum of a finite and small number of exponential terms to the AIMD data, with $I_{j}$ and $z_{j}$ as parameters. In the fit procedure we imposed, as usual, a certain number of constraints, in order to comply with the normalization condition, $\sum_{j} I_{j}=1$, and to enforce the first few odd sum rules dictated by Eq. (10) up to $k-1$, for a given even $k$. Given the specific form of our finite mode expansion, which is infinitely often differentiable except at $t=0$, imposition of sum rules not only ensures that the odd derivatives of $F(Q, t)$ up to order $k-1$ vanish at $t=0$, but that all derivatives up to order $k$ exist and are finite at the origin. From a practical point of view, the number of real and complex terms, and of sum rules to be taken into consideration is essentially dictated by physical considerations and, eventually, by the fit quality achieved with a reasonably low (ideally minimized) number of parameters, as discussed below.

In our previous experience about single-particle correlation functions, we found that a small number of real modes is typically required in high-density states around and above the triple point density of the investigated system, while this number grows with decreasing density. Differently, independently of density, two complex pairs are always required (although no more than two), one of which appears as a true constant feature of the dynamics and is readily identified with the longitudinal excitation. The other pair of complex modes may instead completely change nature with varying density. However, the present case of liquid $\mathrm{Ag}$ belongs to the class of "high-density fluids," so it is worth focusing on our previous findings in such thermodynamic conditions for other fluids, where we found that the second complex pair needed for very satisfactory fits to single-particle functions, like the VAF or $S_{\text {self }}(Q, \omega)$, could be unambiguously identified with the contribution due to shear waves $[23,24,45,46]$. In particular, the multimode analysis of $S_{\text {self }}(Q, \omega)$ of liquid $\mathrm{Au}$ [24] was successfully performed by allowing for two real modes and two complex pairs, with the above phys- 
ical meaning for the complex pairs. So, it seemed to us natural to attempt the same scheme for the possible modes of liquid $\mathrm{Ag}$ in our conditions, since similar processes are expected to characterize the dynamics of the two liquid metals.

A word of caution is necessary, however. As recalled in the mentioned papers, $S_{\text {self }}(Q, \omega)$ is strongly related to the VAF spectrum $Z(\omega)$, i.e., to the density of states of the liquid, even at nonzero $Q$ values. Thus, it is not surprising that both these quantities reveal the presence of transverse excitations. Nonetheless, the situation might be quite different in the case of the total $S(Q, \omega)=S_{\text {dist }}(Q, \omega)+S_{\text {self }}(Q, \omega)$ [or $\left.F(Q, t)\right]$, since the role played by the distinct part of this function is substantially unknown and might have the effect to completely hide, in the total function, what it is instead clearly detected from its self part. In fact, this was exactly the case with $S(Q, \omega)$ of gold, where no low-frequency inelastic features could be observed, not even at high $Q$ values, despite the clear indications suggested by its simulated VAF.

We preliminarily note that the fits were performed to the AIMD $F(Q, t) \mathrm{Ag}$ data up to a maximum time value $t_{\mathrm{R}}=$ $0.5 \mathrm{ps}$, which corresponds to the so-called recurrence time due to the use of periodic boundary conditions in the simulations $[68,69]$. The recurrence time is roughly the maximum time lag beyond which the calculations are no more reliable and typically present spurious oscillations. It is usually identified with the time a density fluctuation takes in propagating over a distance equal to the box length, traveling at the sound speed $c_{\mathrm{s}}$. For a cubic box, it can be evaluated as $t_{\mathrm{R}}=(N / n)^{1 / 3} / c_{\mathrm{s}}$.

Our attempts to fit $F(Q, t)$ with two real modes and two complex pairs failed in the whole $Q$ range. We thus turned to different modelings. In particular, we compared the performances of two fit functions: A one-excitation model as the VE, and a two-excitation model, here referred to as $2 \mathrm{C}$. In the exponential representation, the first includes two real modes (labeled as R1 and R2, and accounting for thermal and structural relaxation $[3,64,66]$, respectively) and one complex pair (labeled as C2 and accounting for sound waves), with three obeyed sum rules. We recall that the last condition ensures the finiteness of the second and fourth frequency moments of $S(Q, \omega)$. The second includes two complex pairs (C2 and $\mathrm{C} 3$ ) and one real mode, with three sum rules obeyed as for the VE fits. In this second model, C2 still stands for the longitudinal component, while $\mathrm{C} 3$ represents the possible transverse contribution. Clearly, as commented about the GH model at the end of Sec. II, the single real mode permitted by the fit algorithm when using this model is to be interpreted as an effective way of accounting for the central part of the AIMD spectra (minimizing the overall number of parameters the fit is able to manage) and cannot be assigned to a specific relaxation process, although it is rather obvious that it must reflect the presence of both thermal and structural effects. It is worth anticipating that for both the $\mathrm{VE}$ and $2 \mathrm{C}$ fits we are going to discuss, the resulting second frequency moment, whose finiteness is enforced by the imposition of the second odd sum rule, turned out to agree, within a few percents, with the theoretical value $k_{\mathrm{B}} T Q^{2} / M$, with $M$ the atomic mass of Ag.

For a better understanding of the following, it is useful to keep in mind the typical behavior of the longitudinal dispersion curve of a liquid (refer, e.g., to Fig. 9 of Ref. [4]). Its main characteristics are its bell-like shape in the range $0<Q<Q_{p}$ and, particularly, the existence of the so-called "sound propagation gap" [70] around $Q_{p}$, where the longitudinal mode $(\mathrm{C} 2)$ frequency $\operatorname{Im} z_{\mathrm{C} 2}=\omega_{\mathrm{s}}$ goes to zero. The arrest of sound propagation around $Q_{p}$ is a general physical property of simple liquids, linked to what de Gennes identified with a spectral narrowing [68]. In the propagation gap region, fits typically become very unstable, whatever model is used and whatever system is considered, due to the difficulties encountered by a fit algorithm when the spectrum, in proximity of $Q_{p}$, tends, as a fact, to include the superposition of multiple central lines (i.e., those foreseen by the real modes of the chosen model plus the two overlapping lines corresponding to an overdamped longitudinal oscillation). For this reason we will not analyze the dynamics in the region around $Q_{p}$. Nevertheless, an attempt to recover some information on the (transverse) collective modes in the gap range, by employing a different approach, will be discussed in the Appendix.

Given these premises, we found that the VE line shape allows for very good fits of the liquid silver AIMD data up to $Q=14 \mathrm{~nm}^{-1}$, which slightly exceeds $Q_{p} / 2$. In such a $Q$ range, the $2 \mathrm{C}$ model provided instead a not significant frequency for the C3 pair. An example of the VE very good performance at such wave vectors is given in Fig. 5, where both the time correlation function and the spectrum are reported, along with the VE fit and its components. The plots in semilogarithmic scale are reported to appreciate the performance of the model in the mentioned range.

Differently, at few $Q$ values above $Q=14 \mathrm{~nm}^{-1}$, i.e., just when $\omega_{\mathrm{s}}(Q)$ starts to decrease after having reached its maximum at $\sim Q_{p} / 2$, we observed that impressively better fits were obtained with the $2 \mathrm{C}$ line shape over the VE one. In particular, the undamped frequency of $\mathrm{C} 3, \Omega_{\mathrm{t}}=$ $\sqrt{\left(\operatorname{Re} z_{\mathrm{C} 3}\right)^{2}+\left(\operatorname{Im} z_{\mathrm{C} 3}\right)^{2}}=\sqrt{z_{\mathrm{t}}^{2}+\omega_{\mathrm{t}}^{2}}$, turned out to be rather similar to the $\Omega_{\mathrm{T}}$ values derived from the transverse current autocorrelation we reported in Ref. [7]. The same superiority of the 2C model was found at almost all $Q$ values beyond the gap, i.e., above $Q=31 \mathrm{~nm}^{-1}$. Examples of the quality of the 2 C fits are shown in Figs. 6 and 7, at two $Q$ values, one smaller and one larger than the longitudinal gap $Q$ range.

However, when $\omega_{\mathrm{s}}$ starts to decrease after its first maximum, the $2 \mathrm{C}$ fit is again unable to reveal a second oscillatory component (C3) and, in fact, a VE modeling provides again a better account of the simulated spectrum. This result is somewhat puzzling: While a one-excitation behavior is reasonable at small $Q$, this is less convincing at the wave-vector values approaching the gap $\left(19<Q<21 \mathrm{~nm}^{-1}\right)$, that is, after that a clear evidence of transverse modes was obtained at a few smaller $Q$ values. Rather than signaling a true change in the physical behavior, this probably reflects the fact that, while decreasing towards zero, $\omega_{\mathrm{s}}$ falls into the typical range of $\omega_{\mathrm{t}}$ and the two excitations overlap each other. Moreover, we think that, in the above mentioned $Q$ range, a strongly damped mode as $\mathrm{C} 3$ becomes indistinguishable, within the fit sensitivity, from a real mode. Therefore, the viscoelastic model shows a better performance simply because it is characterized by a lower number of parameters. A simpler line shape seems to partly solve the problems encountered with the 2C model at those $Q$ values where the longitudinal and 

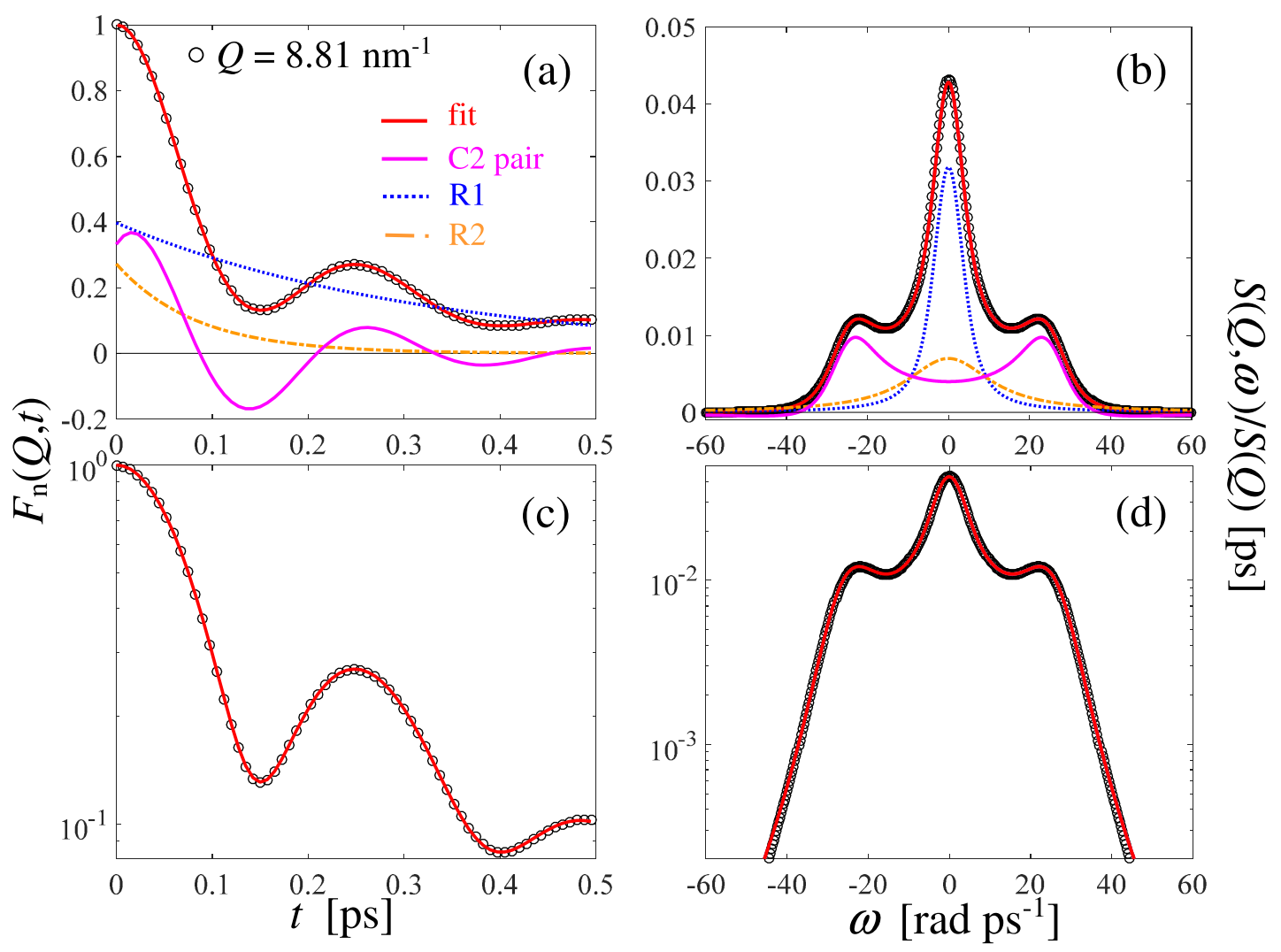

FIG. 5. (a) Simulated intermediate scattering function $F_{\mathrm{n}}(Q, t)$ of liquid $\mathrm{Ag}$ at $Q=8.81 \mathrm{~nm}^{-1}$ (black circles) and VE fit result (red solid curve). The multiexponential fit components are also shown separately and specified in the legend. (b) Corresponding spectrum $S(Q, \omega) / S(Q)$, VE fit result, and fit components, following the same legend of panel (a). Panels (c) and (d) show the time correlation and its spectrum, respectively, on a semilogarithmic scale in order to better appreciate the quality of the fit (red solid curve).

transverse mode frequencies are not largely separated, and both are strongly damped. As a consequence, in a rather wide $Q$ region, including the longitudinal gap, the study of $S(Q, \omega)$ leaves the transverse dynamics substantially undetermined (see, however, the Appendix).

A further comment is worth about Figs. 6(a) and 7(a): There the $\mathrm{C} 3$ component is clearly seen to give a significant contribution to the total function, with an intensity that grows with increasing wave vector. A possible different interpretation of the low-frequency modes relates them to the propagation of thermal waves in the fluid [71,72]. However, the only available results show that their contribution to $S(Q, \omega)$ is nearly negligible and practically constant with varying $Q$ [71]. Therefore, it would be an unjustified hypothesis to associate the $\mathrm{C} 3$ excitation modes to such a phenomenon in our case.

In Fig. 8(b) we report our best determination of the dispersion curve of liquid Ag from AIMD data obtained by performing the above described multimode fit analysis, with three obeyed sum rules. The upper, bell-shaped, branch is the longitudinal mode dispersion $\omega_{\mathrm{s}}(Q)$. For this mode, the low- $Q$ hydrodynamic linear behavior is also shown, together with the $\mathrm{GH}$ experimental results in the limited $Q$ range of the experiment. Exactly as it happened for gold, the GH model tends to slightly underestimate the frequency at the highest experimental wave vectors. Nonetheless, it captures, within the experimental errors, the low- $Q$ longitudinal dynamics very well. In the $Q$ regions where transverse modes were detectable, i.e., when the $2 \mathrm{C}$ model was indisputably superior to the $\mathrm{VE}$ one, the dispersion curve also shows a lower frequency branch, $\omega_{\mathrm{t}}(Q)$. As explained, we report in Fig. 8(b) the $\omega_{\mathrm{t}}$ values only at those $Q$ where we are confident that shear waves also give a significant fingerprint in $F(Q, t)$ of liquid silver, and fit algorithms clearly distinguish between the longitudinal and transverse component. The latter branch displays a somewhat smaller accuracy and less smooth behavior as a direct consequence of the fact that the dynamics of shear modes is at the limits of detectability from a quantity like $F(Q, t)$ or, equivalently, $S(Q, \omega)$.

As done in other papers [24,73], the simultaneous analysis of the VAF spectrum $Z(\omega)$ and the dispersion curve can further support the validity of the results obtained from the fits. As previously mentioned, $Z(\omega)$ has peaks or (sometimes feeble) shoulders where vibrational state frequencies occur more often with varying $Q$, i.e., as already stated, it embodies the density of states of the liquid. Its behavior can help not only in confirming the presence of propagating modes of both longitudinal and transverse nature, but also in establishing which excitations give rise to flat branches in the dispersion curve (see, e.g., Fig. 4 of Ref. [73] or Fig. 7 of Ref. [24]). In fact, a weakly dispersive mode should leave a strong signature in $Z(\omega)$, analogously to what is referred to as a van Hove singularity in solid state phonon dynamics [74]. For this reason, we plot also in Fig. 8(a) the spectrum of the AIMD normalized VAF on the same frequency scale used to 

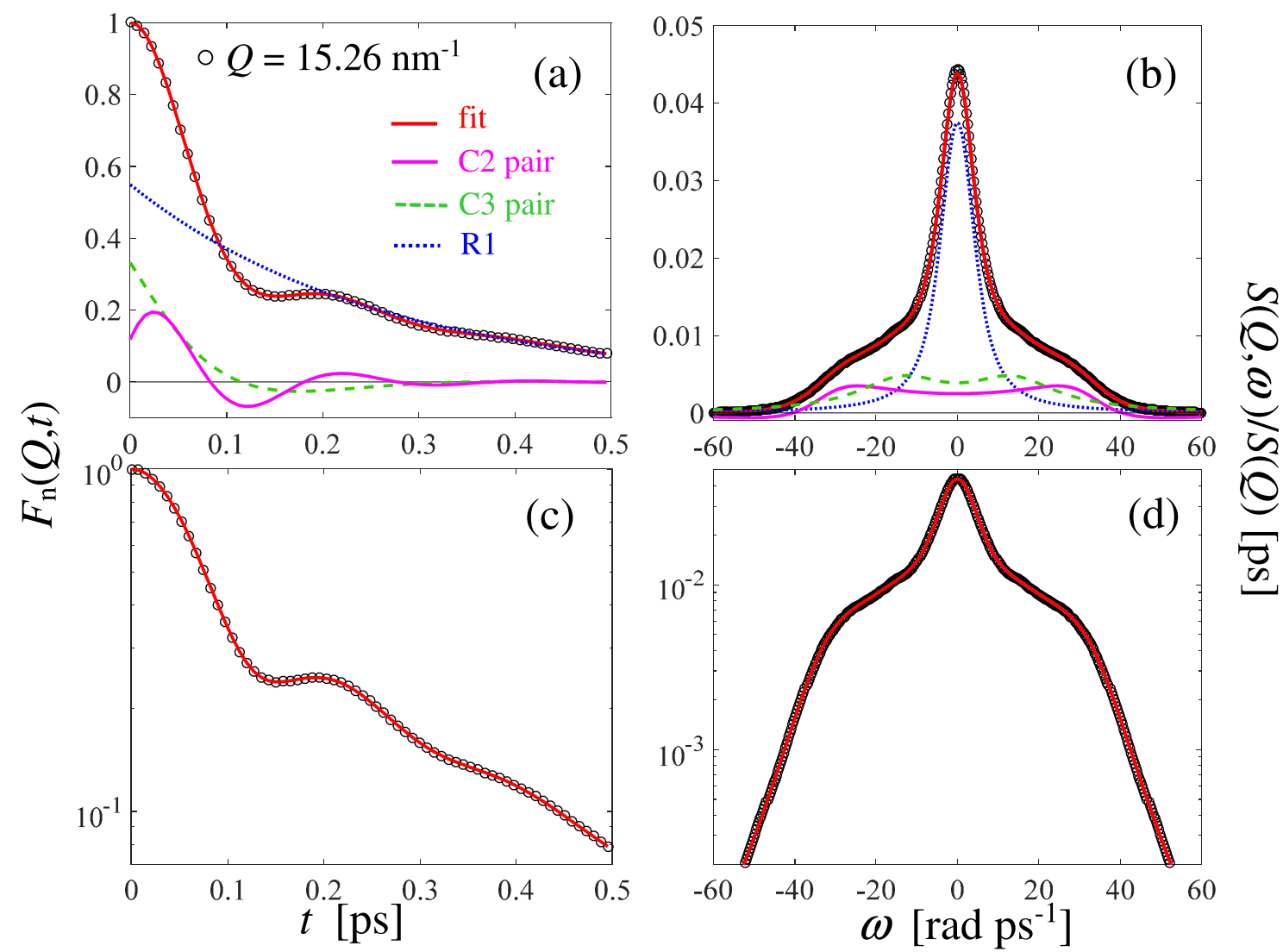

FIG. 6. (a) Simulated intermediate scattering function $F_{\mathrm{n}}(Q, t)$ of liquid $\mathrm{Ag}$ at $Q=15.26 \mathrm{~nm}^{-1}$ (black circles) and $2 \mathrm{C}$ fit result (red solid curve). The multiexponential fit components are also shown separately, and specified in the legend. (b) Corresponding spectrum $S(Q, \omega) / S(Q)$, $2 \mathrm{C}$ fit result, and fit components, following the same legend of panel (a). Panels (c) and (d) show the correlation and its spectrum, respectively, on a semilogarithmic scale in order to better appreciate the quality of the fit (red solid curve).

plot the dispersion curve of Fig. 8(b). As usual, it is seen that $Z(\omega)$ slightly inflates in rough correspondence with the maximum of $\omega_{\mathrm{s}}(Q)$, and peaks around the, almost constant in $Q$, value of $\omega_{\mathrm{t}}$. These observations can be quantitatively confirmed by determining the exponential decomposition of the AIMD VAF which, similarly to Eq. (8) for $F(Q, t)$, admits a multiexponential expansion of the kind

$$
Z_{\mathrm{n}}(t)=Z(t) / Z(0)=\sum_{j=1}^{\infty} A_{j} \exp \left(w_{j}|t|\right) .
$$

In analogy with the case of the VAF of Au [24], we found a very good account of the VAF of Ag by performing fits including two complex pairs (labeled as $\mathrm{C}_{Z}$ and $\mathrm{C}_{Z}$ ) and one real mode. The complex components represent, as usual, the contributions to $Z(t)$ due to the longitudinal and transverse dynamics. The subscript $Z$ is used to clarify that these complex pairs are not to be identified with those (C2 and $\mathrm{C} 3$ ) found for $F(Q, t)$, despite that they are obviously the fingerprint in the VAF of the same collective processes. The real mode, labeled as $\mathrm{R} 2_{Z}$, is the only one that survives in the VAF of the two real modes usually necessary, along with the complex pairs, for the description of $F_{\text {self }}(Q, t)$ of a liquid metal. In fact, as shown in the case of $\mathrm{Au}$ [24], $Z(t)$ does not contain the self-diffusion Fick's mode of $F_{\text {self }}(Q, t)$.

The quality of the fit to the VAF of Ag is shown in Fig. 9, along with the individual real and complex components of the model. The negative amplitude of $\mathrm{R} 2_{Z}$ is a straightforward consequence of the fact that $Z(t)$ is related to the second time derivative of $F_{\text {self }}(Q, t)$, in the $Q \rightarrow 0$ limit. Thus, a positive real contribution in $F_{\text {self }}(Q, t)$ unavoidably translates into a negative real component in $Z(t)$ (see the Appendix). The above described fit-based analysis of the VAF of Ag locates the frequency positions of the maximum and of the longitudinal shoulder of the VAF spectrum at $\omega=\operatorname{Im} w_{\mathrm{C}_{3}}=13.0 \mathrm{rad}$ $\mathrm{ps}^{-1}$ and $\omega=\operatorname{Im} w_{\mathrm{C}_{2}}=31.1 \mathrm{rad} \mathrm{ps}^{-1}$, respectively. Corresponding dampings of the same modes are $-\operatorname{Re} w_{\mathrm{C}_{3}}=13.1$ $\mathrm{ps}^{-1}$ and $-\operatorname{Re} w_{\mathrm{C}_{2}}=11.4 \mathrm{ps}^{-1}$, which give an idea of the frequency bands that the density of states covers around these two characteristic frequencies of the dynamics of Ag. These frequencies were shown also in Fig. 8(a) as dashed green $\left(\operatorname{Im} w_{\mathrm{C}_{Z}}\right)$ and dashed pink $\left(\operatorname{Im} w_{\mathrm{C}_{Z}}\right)$ straight lines in order to show their evident correspondence with the flat zones of the dispersion curve. The dynamical picture is therefore more than clear.

Finally, for completeness, in Fig. 10 we show the results for the $Q$ dependence of the damping $-\operatorname{Re} z_{\mathrm{C} 2}=z_{\mathrm{s}}$ and of the undamped frequency $\Omega_{\mathrm{s}}=\sqrt{\omega_{\mathrm{s}}^{2}+z_{\mathrm{s}}^{2}}$ of the longitudinal mode of $F(Q, t)$, which resemble very much those of gold and, qualitatively, those of insulating liquids as $\mathrm{CO}_{2}$ [64]. The undamped frequency $\Omega_{\mathrm{t}}$ and damping $-\operatorname{Re} z_{\mathrm{C} 3}=z_{\mathrm{t}}$ of the transverse mode are also reported in the figure, along with the curve obtained by locating the maxima of the AIMD transverse autocorrelation spectra $C_{\mathrm{T}}(Q, \omega)$. 

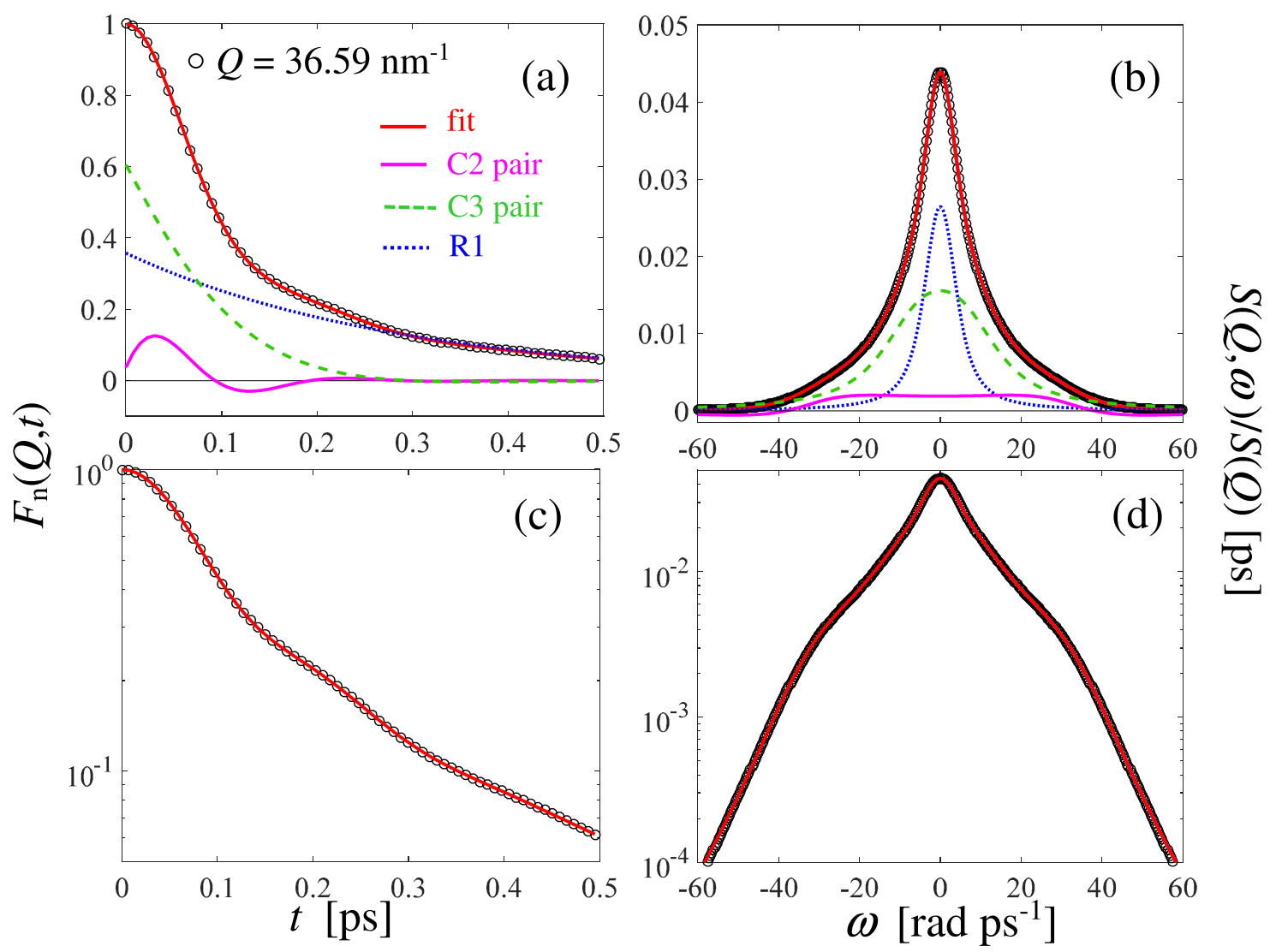

FIG. 7. Same as Fig. 6 at $Q=36.59 \mathrm{~nm}^{-1}$, i.e., after the propagation gap of the longitudinal modes.
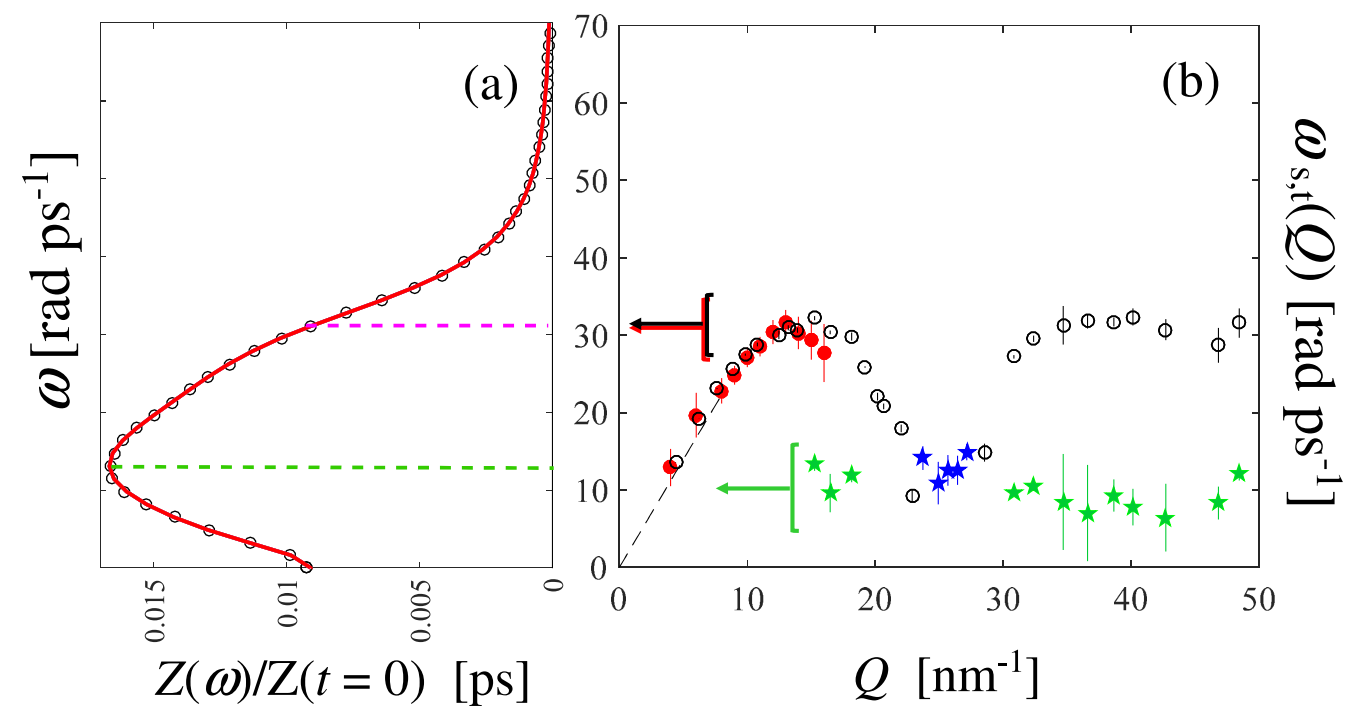

FIG. 8. (a) VAF spectrum (liquid silver density of states) from AIMD simulations (open circles). The dashed green and pink lines indicate the frequencies $\operatorname{Im}\left[w_{\mathrm{C}_{Z}}\right]$ and $\operatorname{Im}\left[w_{\mathrm{C} 2_{Z}}\right]$, respectively, obtained from the multiexponential fit of Eq. (11) to the normalized VAF, discussed in the text and displayed in detail in Fig. 9. (b) Dispersion curve (black circles with error bars) resulting from the fits of the simulated AIMD $F(Q, t)$. The red full circles are the corresponding experimental results obtained by means of GH fits to the neutron data. The straight black dashed line is the linearized hydrodynamics prescription. Data are missing around the position of the main maximum in the static structure factor due to the instability of the fit results in such a wave-vector region. Green stars are the transverse-like frequencies obtained according to model $2 \mathrm{C}$, when successful. The blue stars in the transverse branch of the dispersion curve were obtained as detailed in the Appendix, taking into consideration the longitudinal current behavior in the longitudinal gap region. Note the physical link between the branches of the dispersion curve in panel (b), and the more or less visible features of $Z(\omega)$ in panel (a). The frequency intervals where the branches of the dispersion curve have a nearly horizontal tangent are approximately indicated by the colored brackets in panel (b). The corresponding arrows (black/red for longitudinal, and green for transverse modes) are used to highlight their clear agreement with the dashed lines in panel (a), locating the above quoted frequencies of $Z(\omega)$. 

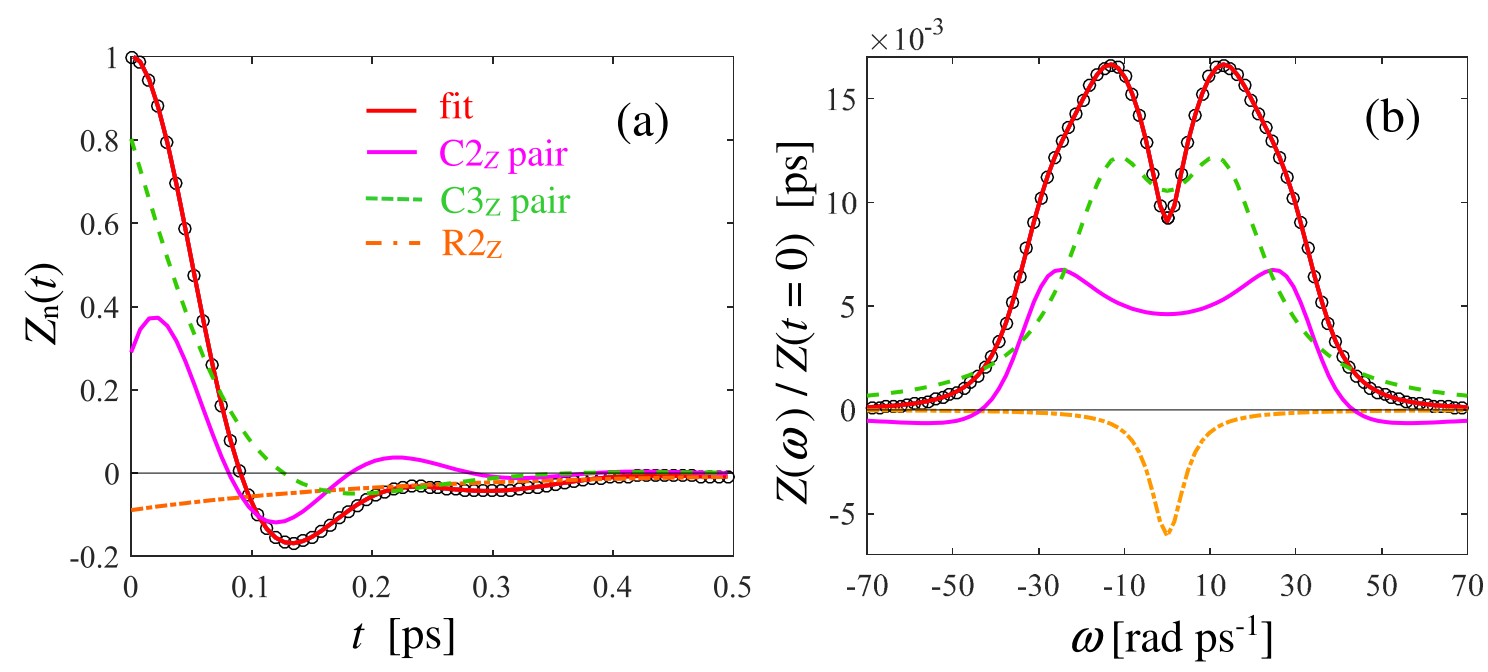

FIG. 9. (a) Normalized velocity autocorrelation function $Z_{\mathrm{n}}(t)$ of liquid $\mathrm{Ag}$ (black circles) and multiexponential fit result (red solid curve). The fit components are also shown separately, and detailed in the legend. (b) Corresponding spectrum $Z(\omega) / Z(t=0)$, fit result, and individual components, following the same legend of panel (a).

\section{CONCLUSIONS}

In this work we addressed several important aspects concerning the dynamics of the liquid state, and in particular of liquid metals. First, we presented a very detailed analysis of a NBS experiment performed on BRISP at the ILL, aimed at reaching knowledge about the dynamic behavior of liquid Ag, for comparison with previous similar measurements on $\mathrm{Au}$

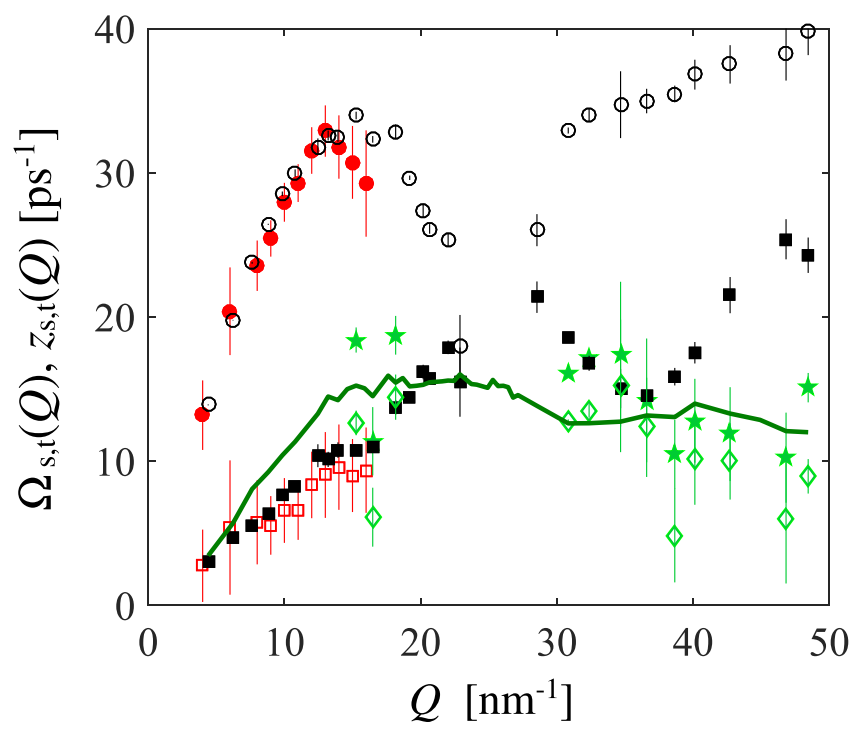

FIG. 10. $Q$ dependence of the undamped frequency $\Omega_{\mathrm{s}}$ (black circles with error bars) and the damping $z_{\mathrm{s}}$ (black full squares with error bars) of the longitudinal modes obtained from the fits to the AIMD $F(Q, t)$ as explained in the text. The same, $\Omega_{\mathrm{t}}$ (green full stars with error bars) and $z_{\mathrm{t}}$ (green empty diamonds with error bars), is shown for the transverse modes when unambiguously determined by the fits (see text). The full red circles and open red squares are the corresponding experimental quantities resulting from the GH fit to the neutron spectra. The $Q$ dependence of the frequency of the maximum in the AIMD transverse current autocorrelation spectrum $C_{\mathrm{T}}(Q, \omega)$ is also displayed as a dark green solid curve. which did not reveal, differently from other metals, evidence of shear waves from the spectral analysis of the dynamic structure factor $S(Q, \omega)$. Despite the difficulties posed by experiments requiring high temperature and on highly absorbing samples, the evident quality of the Ag experimental results confirms the very good performances of BRISP and the importance of a careful data analysis of any inelastic neutron-scattering experiment, especially those on liquids and in the NBS $Q$ range. Within the experimental uncertainties, we were able (as for $\mathrm{Au}$ ) to characterize very well the behavior of longitudinal modes through a $\mathrm{GH}$ modeling of the measured spectra, but no other feature could be revealed through other more complex modelings of the experimental line shape within the errors. However, besides reproducing the static structure [7], we clearly showed that our AIMD simulations also turn out to describe very well the experimental results for $S(Q, \omega)$ in the available $Q$ range. Such a validation opened the way to a detailed analysis of the AIMD results for $F(Q, t)$ in the much more extended $Q$ range that simulations allow to probe compared to experiments.

In the present case of $\mathrm{Ag}$, we took the chance to further test the performance of the multiexponential decomposition method of time correlation functions also for a collective quantity like the intermediate scattering function. Given the quality of all the presented fits, and the physical sensibility of the results, we confirm its success for correlation functions as the VAF, $F_{\text {self }}(Q, t)$, and $F(Q, t)$.

From a physical point of view, we deduced what follows. Differently from the case of $\mathrm{Au}$, here we can firmly conclude that transverse-like excitations are actually detectable from the simulated $F(Q, t)$, although with some inaccuracies and only at rather high $Q$ values. However, this result does not necessarily imply that $\mathrm{Au}$ and $\mathrm{Ag}$ have substantially different dynamical behaviors. It could well be possible that transverse modes, which in general produce at most very weak features in $S(Q, \omega)$, are in the two metals just below and above the detectability threshold, respectively. In such a situation the noise level can affect largely the final results, and a progress in the simulation methods might also contribute an explanation 
of seemingly different outcomes, without forcedly signaling true significant differences in the intrinsic dynamics of the two systems. Thus, group IB elements may actually turn out not to be an exception, as far as the detectability of shear waves from simulated $S(Q, \omega)$ is concerned: The cases to investigate more deeply remaining however $\mathrm{Au}$ and $\mathrm{Cu}$.

It is well known that shear waves show up at nonzero $Q$ values, but in the present case of Ag they start to be detectable in $F(Q, t)$ or $S(Q, \omega)$ at higher wave vectors than those reported in works on different liquid systems. Such a retarded visibility $\left(Q \approx 15 \mathrm{~nm}^{-1}\right)$ is of course a consequence of our one-excitation description at lower wave vectors where the second complex pair of model $2 \mathrm{C}$ was found to be physically unjustified, with close to zero and nonsignificant frequency, i.e., playing the role of simply mimicking the second central Lorentzian of the VE model. Vice versa, we believe that at $Q$ values where a transverse dynamics has clearly set on, but strongly damped modes overlap each other (as it happens when $\omega_{\mathrm{s}}$ starts to decrease), the fit algorithms cannot resolve multiple, central and close to central, lines contributing to $S(Q, \omega)$, unless by reducing the number of free parameters. In such $Q$ ranges $\left(19<Q<21 \mathrm{~nm}^{-1}\right)$, where the $2 \mathrm{C}$ model fails differently from the VE one, we believe that it is the VE model which, due to its simpler structure, hides part of the real dynamics. The problem clearly lies in the less structured shape of $S(Q, \omega)$ as the longitudinal gap is approached.

In the end, we deduced that a full characterization of the collective dynamics based on the analysis of simulated $F(Q, t)$ or $S(Q, \omega)$ is not at all trivial, and can reach a scientific significance only when imposing some mandatory physical constraints, and only when the frequency of the longitudinal mode at least doubles the transverse one.

In some respects, however, the case of group IB elements apparently differs from that of other metals, where a transverse dynamics was deduced even from the experimental $S(Q, \omega)$. In fact, the situation for IB metals is still unsettled if one merely refers to experimental $S(Q, \omega)$ data, since these are unable to reveal shear modes within the errors: A still unsolved case being also that of copper, as elucidated in the Appendix. As far as we can observe at a purely experimental level, no shear waves have been determined when physically constrained fits to measured data ( $\mathrm{Ag}, \mathrm{Au}$ ) have been performed: This leaves an open question on the experimental detectability of a low- $Q$ transverse dynamics in simple systems as liquid metals [5,8-11].

Conversely, by focusing on simulated and smoother $F(Q, t)$ or $S(Q, \omega)$ results it has been possible to obtain reliable evidence of a two-excitation dynamics in $\mathrm{Ag}$ in a limited $Q$ range. We showed, however, that the transverse branch of the dispersion curve can be hard to determine if fundamental physical properties of $S(Q, \omega)$ are to be obeyed. This holds true, in particular, around the beginning and the end of the $Q$ range between 0 and $Q_{p}$.

Nonetheless, the main message of this work does not exclusively focus on the detectability of transverse modes in liquids or on the comparison of different systems. Here we wished to show that reliable studies of the transverse-like dynamics of whatever liquid system require very high-quality data, which justify the efforts for a constant improvement of simulation and experimental methods, along with the use of theoretically well-founded models. Looking at the presence of "peaks" in $S(Q, \omega)$ or in the longitudinal current-current correlation spectrum, without a theoretical basis and without a clear physical self-consistency of the used fit algorithms, can lead to misinterpretations.

From another point of view, our work confirms the importance of looking at as many correlation functions as possible, although not all experimentally accessible, to get insight in the dynamical behavior of a liquid. Thus, it is clearly shown that the capabilities of simulations and the interpretation tools they provide progressively approach, and sometimes equal or overtake, the experimental possibilities through a continuous improvement of modelings, methods, and computational power.

\section{ACKNOWLEDGMENTS}

The authors wish to thank Francesco Sacchetti and Simone Aisa of the Physics and Geology Department of the University of Perugia (Italy) for providing us with the sample container. We wish to express our gratitude to Stefano Bellissima for his significant contribution in setting up and testing the multimode analysis of correlation functions of several fluids. We do thank the ILL staff for helping us conducting this experiment. This research was funded by Ente Cassa di Risparmio di Firenze (Grant No. 2016-0866) and Ministero dell'Istruzione dell'Università e della Ricerca Italiano (Grant No. PRIN20172017Z55KCW), and by Ministerio de Economía y Competitividad (Project PGC2018-093745-B-I00), partially funded by European Regional Development Fund funds, and Junta de Castilla y León (project VA124G18).

\section{APPENDIX A: ANALYZING THE LONGITUDINAL CURRENT-CURRENT SPECTRUM}

Here we discuss what can be obtained if, in place of $S(Q, \omega)$, one carries out a multimode analysis of the longitudinal current-current spectrum $C_{\mathrm{L}}(Q, \omega)$ to investigate the transverse dynamics of a liquid, taking into account that both quantities carry, in principle, the same dynamical information.

Preliminarily, we consider the case of a generic autocorrelation function $c(t)$ related to the second time derivative of another (normalized) autocorrelation $b(t)$, e.g., $c(t)=$ $-\ddot{b}(t)$, whose spectrum is $c(\omega)=\frac{1}{2 \pi} \int c(t) \exp (-i \omega t) d t=$ $\omega^{2} b(\omega)$. By using the exponential representation of $b(t)=$ $\sum_{j} I_{j} \exp \left(z_{j} t\right)$ at $t>0$, direct double differentiation of course leads to

$$
c(t)=\sum_{j}\left(-I_{j} z_{j}^{2}\right) e^{z_{j} t},
$$

so that the following relations hold when switching to $\omega$ space:

$$
\begin{aligned}
c(\omega) & =\omega^{2} b(\omega) \\
& =\omega^{2} \sum_{j} \frac{I_{j}}{\pi}\left[\frac{\left(-z_{j}\right)}{\omega^{2}+z_{j}^{2}}\right] \\
& =\sum_{j} \frac{I_{j}}{\pi}\left[\frac{\left(-z_{j}\right)\left(\omega^{2}+z_{j}^{2}-z_{j}^{2}\right)}{\omega^{2}+z_{j}^{2}}\right]
\end{aligned}
$$




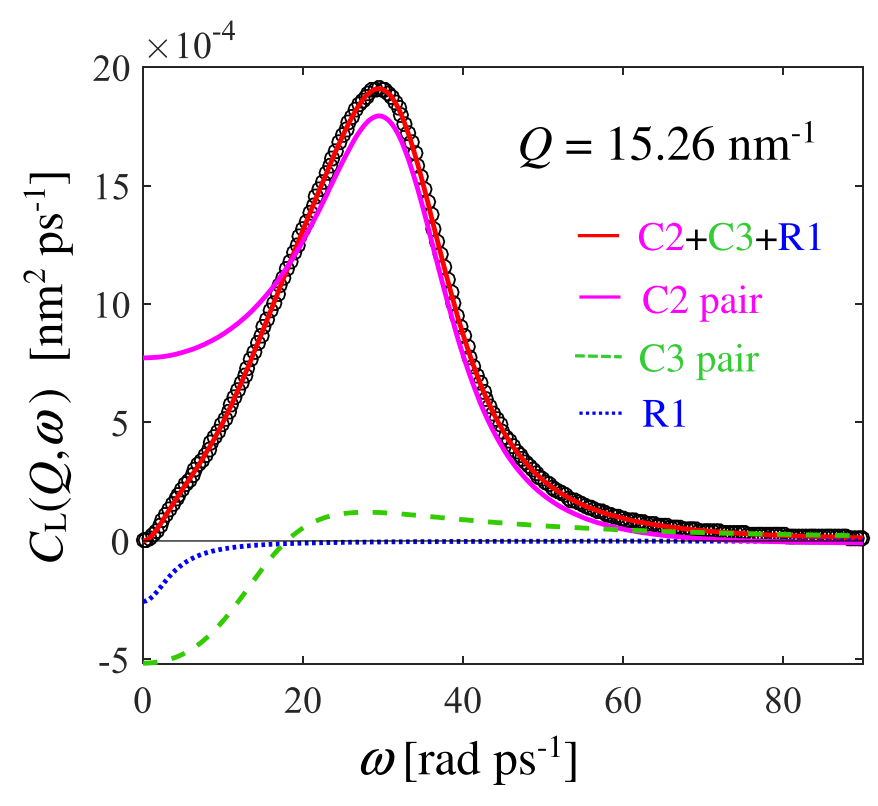

FIG. 11. Longitudinal current-current spectrum derived from the AIMD $S(Q, \omega)$ of liquid Ag (black circles). The chosen $Q$ belongs to a wave-vector range where shear modes were clearly detected, as shown in Fig. 6. The individual contributions of the modes to $C_{\mathrm{L}}(Q, \omega)$ were calculated according to Eq. (A4), using the $I_{j}$ and $z_{j}$ values resulting from the $2 \mathrm{C}$ fits discussed in Sec. IV. The same labeling, curve style, and color code of Fig. 6 was used. The red solid curve corresponds to the resummation of the various components.

$$
\begin{aligned}
& =\sum_{j} \frac{I_{j}}{\pi}\left[-z_{j}+\frac{z_{j}^{3}}{\omega^{2}+z_{j}^{2}}\right] \\
& =\sum_{j} \frac{\left(-I_{j} z_{j}^{2}\right)}{\pi}\left[\frac{\left(-z_{j}\right)}{\omega^{2}+z_{j}^{2}}\right],
\end{aligned}
$$

where the first term in the last but one member of Eq. (A2) vanishes due to the first odd sum rule, $\sum_{j} I_{j} z_{j}=0$. In this way a constant term in the $j$ th spectral mode, which would prevent it from decaying to zero and make it nonintegrable, is no longer present. The notation used in the last member of the above equation is simply meant to help recalling that for real modes $-z_{j}$ is a positive quantity, and that the amplitudes $I_{j}$ in $b(\omega)$ change to $\left(-I_{j} z_{j}^{2}\right)$ in $c(\omega)$. Consequently, the amplitudes of the real components of $c(\omega)$ are opposite in sign with respect to the corresponding ones in $b(\omega)$. The sign of the total contribution to $c(\omega)$ of a complex pair, with varying $\omega$, is less trivial to deduce, since it depends on the possible combinations of the real and imaginary parts of both $I_{j}$ and $z_{j}$ : Combinations that are not bounded to take the same sign with varying the complex pair of the scheme, the only condition being $\operatorname{Re} z_{j}<0$ for all modes. Summarizing, the scheme of the modes for the two time autocorrelations is exactly the same, with identical frequencies and dampings. Only the amplitudes of the modes change when switching between $b(t)$ and $c(t)$, and their spectra.

A well-known example in which two physically significant autocorrelations are linked by a double time differentiation is

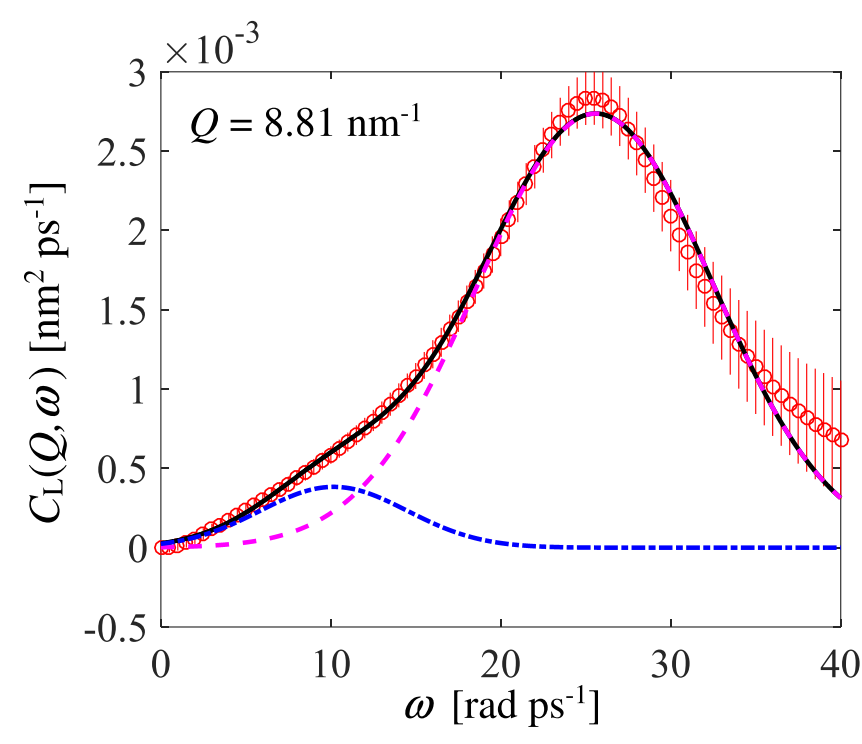

FIG. 12. Longitudinal current-current spectrum derived from our VE description of the AIMD liquid Ag data (red circles). The chosen $Q$ belongs to a wave vector range where transverse waves cannot yet be detected from reliable analyses of the dynamic structure of $\mathrm{Ag}$, as commented about Fig. 5. In order to "simulate" an experimental condition, we considered the resolution broadening of the x-ray measurements on $\mathrm{Cu}$ [12]. A two-Gaussian fit to the experimentallike $C_{\mathrm{L}}(Q, \omega)$ (black solid curve) suggests also an inexistent, by construction, low-frequency mode (blue chain curve), besides providing an estimate of the frequency of the longitudinal excitation (pink dashed curve).

just the case of $F(Q, t)$ and $C_{\mathrm{L}}(Q, t)$, in fact [3],

$$
C_{\mathrm{L}}(Q, t)=-\frac{1}{Q^{2}} \ddot{F}(Q, t) .
$$

Consequently, given the time representation of $F(Q, t)$ in Eq. (8), the above formalism leads to

$$
C_{\mathrm{L}}(Q, \omega)=\frac{S(Q)}{Q^{2}} \sum_{j} \frac{\left(-I_{j} z_{j}^{2}\right)}{\pi}\left[\frac{\left(-z_{j}\right)}{\omega^{2}+z_{j}^{2}}\right] .
$$

Therefore, once the modes of $S(Q, \omega)$ are known, we can readily find the correct, theoretically valid, multimode decomposition of $C_{\mathrm{L}}(Q, \omega)$ by simply exploiting Eq. (A4). Note that, since the amplitudes of the modes of $C_{\mathrm{L}}(Q, \omega)$ contain a factor $z_{i}^{2}$, the relative weight of the modes obviously changes drastically in switching from $S(Q, \omega)$ to $C_{\mathrm{L}}(Q, \omega)$. Moreover, as described below, transverse modes turn out to give a negative contribution to $C_{\mathrm{L}}(Q, \omega)$ at low frequency, making the latter function unable to display features related to shear waves, at least in liquid metals.

In Fig. 11 we show the longitudinal current-current spectrum of silver at a $Q$ value of $15.26 \mathrm{~nm}^{-1}$ where the analysis of the simulated $S(Q, \omega)$ discussed in Sec. IV revealed the presence of transverse-like excitations. The individual contributions of the various modes to the longitudinal current are also plotted, using of course the same labeling and color codes used for those of $S(Q, \omega)$ in Fig. 6. As it is seen from the figure, $C_{\mathrm{L}}(Q, \omega)$ is obviously dominated by the longitudinal component and displays no low-frequency feature attributable 

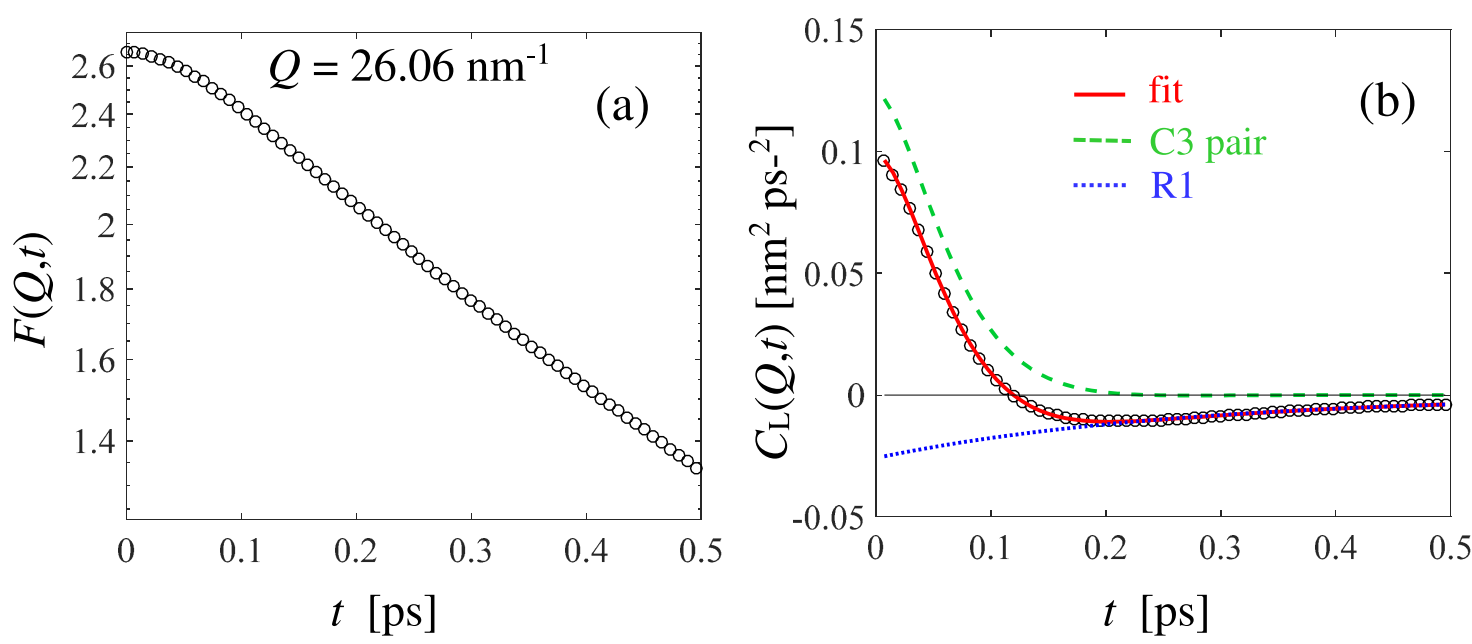

FIG. 13. (a) AIMD intermediate scattering function at a $Q$ value belonging to the longitudinal gap range around $Q_{p}$. Note that no oscillation of the function is visible, not even in semilogarithmic scale. (b) Longitudinal current autocorrelation function (black circles) at the same wave-vector value of panel (a). The global fit to $C_{\mathrm{L}}(Q, t)$ (red solid curve) was obtained with a model that accounts for one complex pair (the transverse-like one, dashed green curve) and one real mode (dotted blue curve).

to shear waves, especially at the frequency value (around $13 \mathrm{rad} \mathrm{ps}^{-1}$ ) determined, at the mentioned $Q$, from the $2 \mathrm{C}$ fit shown in Fig. 6. Shear modes of course influence the overall behavior of $C_{\mathrm{L}}(Q, \omega)$, but it is clear that their frequency cannot be determined by simply looking for some extra intensity in the longitudinal current spectrum, and trying in some way to locate its frequency. In fact, their true contribution to $C_{\mathrm{L}}(Q, \omega)$ is shown to be predominantly negative, and to mainly counteract the high positive contribution of $\mathrm{C} 2$ just in the frequency range (below $20 \mathrm{rad} \mathrm{ps}^{-1}$ ) where the transverse excitation has been found. Notably, such a behavior of the transverselike component, in a correct multi-Lorentzian representation of $C_{\mathrm{L}}(Q, \omega)$, is shown to have the opposite effect than that supposed in the literature, i.e., it tends to deplete the total signal rather than giving rise to positive contributions in the spectrum.

As a consequence, (1) the low-frequency shape of the total $C_{\mathrm{L}}(Q, \omega)$ of a simple liquid metal has no special physical meaning, and simply originates from the interplay of the various modes, and from their respective dependence on $\omega$ and (2) the presence of a shear component actually tends to make the low-frequency longitudinal current spectrum smoother and featureless than in the case of an either too weak or absent transverse-like dynamics. This fact will become evident below, particularly when comparing the shape of $C_{\mathrm{L}}(Q, \omega)$ in the presence (Fig. 11) or absence (Fig. 12) of transverse-like modes. Experimental resolution effects taken into account in the following discussion, and considered for Fig. 12, can be easily demonstrated to be irrelevant as far as the conclusions about the role played by shear waves in $C_{\mathrm{L}}(Q, \omega)$ are concerned.

We further note that, due to the rather high ratio of longitudinal to transverse frequencies typical of liquid metals, multiplication of the amplitudes by $-z_{j}^{2}$ enhances the longitudinal modes with respect to the transverse ones, making $C_{\mathrm{L}}(Q, \omega)$ not convenient for studies of the transverse dynamics.

Finally, as anticipated in the Introduction, we show that by relaxing the compliance with the general theoretical
multi-Lorentzian prescription for $S(Q, \omega)$ (or, equivalently, for $\left.C_{\mathrm{L}}(Q, \omega)\right)$ one can erroneously find low-frequency features that actually are not the physical evidence of a transverse dynamics. To do so, we consider a $Q$ region where we clearly showed that shear waves in Ag cannot yet be detected from $S(Q, \omega)$ or $C_{\mathrm{L}}(Q, \omega)\left(Q<Q_{p} / 2\right)$ and where a VE modeling of the intermediate scattering function perfectly describes the simulation data. From the VE fit parameters we can of course calculate the resolution-free $S(Q, \omega)$ of Eq. (9) which, by construction of the VE model, contains exclusively the longitudinal excitation. In order to take into account the typical resolution effects of an experiment, we convoluted the result with the resolution function of the x-ray measurements on $\mathrm{Cu}$ [12] and calculated an experimental-like $C_{\mathrm{L}}(Q, \omega)$. Following the analysis of Ref. [12], we then performed a two-Gaussian fit of the resulting function, shown in Fig. 12 for the $Q=8.81 \mathrm{~nm}^{-1}$ case as an example. Expectedly, the fit locates a second component in $C_{\mathrm{L}}(Q, \omega)$ at low transverse-like frequencies that were however excluded from the beginning, i.e., by the original VE modeling of the spectral line shape. We find this a rather convincing example that no reliable detection of shear waves can be claimed by performing analyses like the one reported for $\mathrm{Cu}$ and other metals [12].

Nevertheless, there still is a situation where the analysis of the longitudinal current autocorrelation can be of some utility. In fact, within the $Q$ region where the sound wave propagation is arrested and its frequency is zero, the transverse excitation is no longer hidden by the longitudinal one. This is better appreciated in the time domain, where $F(Q, t)$ displays no visible oscillations but $C_{\mathrm{L}}(Q, t)$ does show one, though strongly damped. In Fig. 13 we show both functions for the $Q=26.06 \mathrm{~nm}^{-1}$ case. For the reasons explained in Sec. IV, it is quite difficult to use, in the gap, the fit models already applied outside the gap $Q$ range. However, a simplified, somewhat rough, model turns out to be applicable and is able to provide reasonable information on the transverse component. Such a model is composed of one real mode only plus one 
pair of complex ones. With such a model the possibility of an accurate description of the real-mode components (which we know to be more than one) is abandoned, but the only remaining complex pair contribution can be confidently identified with the transverse excitation. Thus, while in the gap region we are still unable to characterize the overdamped (i.e., nonpropagating) longitudinal modes, we can still obtain approximate but reasonable values for $\omega_{\mathrm{t}}$ and $z_{\mathrm{t}}$. In this way we tentatively added four points to the transverse dispersion curve of Fig. 8(b) already determined from fits to $F(Q, t)$. By doing so, we see that the newly found values are in quite reasonable agreement with the overall $Q$ dependence of the transverse excitation frequencies. The results reported in this Appendix confirm that the possibility of determining the $Q$ behavior of the collective modes via the longitudinal current autocorrelation function or spectrum is essentially dependent on the coexistence or not of both longitudinal and transverse modes in the same frequency range.
[1] N. H. March, Liquid Metals (Cambridge University Press, Cambridge, 1990).

[2] W. Montfrooij and I. de Schepper, Excitations in Simple Liquids, Liquid Metals and Superfluids (Oxford University Press, New York, 2010).

[3] U. Balucani and M. Zoppi, Dynamics of the Liquid State (Clarendon, Oxford, 1994).

[4] E. Guarini, U. Bafile, F. Barocchi, A. De Francesco, E. Farhi, F. Formisano, A. Laloni, A. Orecchini, A. Polidori, M. Puglini, and F. Sacchetti, Dynamics of liquid Au from neutron Brillouin scattering and ab initio simulations: Analogies in the behavior of metallic and insulating liquids, Phys. Rev. B 88, 104201 (2013)

[5] M. Zanatta, F. Sacchetti, E. Guarini, A. Orecchini, A. Paciaroni, L. Sani, and C. Petrillo, Collective Ion Dynamics in Liquid Zinc: Evidence for Complex Dynamics in a Non-Free-Electron Liquid Metal, Phys. Rev. Lett. 114, 187801 (2015).

[6] T. Scopigno and G. Ruocco, Microscopic dynamics in liquid metals: The experimental point of view, Rev. Mod. Phys. 77, 881 (2005).

[7] B. G. del Rio, D. J. González, and L. E. González, An ab initio study of the structure and atomic transport in bulk liquid Ag and its liquid-vapor interface, Phys. Fluids 28, 107105 (2016).

[8] S. Hosokawa, M. Inui, Y. Kajihara, K. Matsuda, T. Ichitsubo, W.-C. Pilgrim, H. Sinn, L. E. González, D. J. González, S. Tsutsui, and A. Q. R. Baron, Transverse Acoustic Excitations in Liquid Ga, Phys. Rev. Lett. 102, 105502 (2009).

[9] V. M. Giordano and G. Monaco, Fingerprints of order and disorder on the high-frequency dynamics of liquids, Proc. Natl. Acad. Sci. USA 107, 21985 (2010).

[10] V. M. Giordano and G. Monaco, Inelastic x-ray scattering study of liquid Ga: Implications for the short-range order, Phys. Rev. B 84, 052201 (2011).

[11] S. Hosokawa, S. Munejiri, M. Inui, Y. Kajihara, W.-C. Pilgrim, Y. Ohmasa, S. Tsutsui, A. Q. R. Baron, F. Shimojo, and K. Hoshino, Transverse excitations in liquid Sn, J. Phys.: Condens. Matter 25, 112101 (2013).

[12] S. Hosokawa, M. Inui, Y. Kajihara, S. Tsutsui, and A. Q. R. Baron, Transverse excitations in liquid $\mathrm{Fe}, \mathrm{Cu}$ and $\mathrm{Zn}$, J. Phys.: Condens. Matter 27, 194104 (2015).

[13] M. Sampoli, G. Ruocco, and F. Sette, Mixing of Longitudinal and Transverse Dynamics in Liquid Water, Phys. Rev. Lett. 79, 1678 (1997).

[14] A. Cunsolo, C. N. Kodituwakku, F. Bencivenga, M. Frontzek, B. M. Leu, and A. H. Said, Transverse dynamics of water across the melting point: A parallel neutron and x-ray, inelastic scattering study, Phys. Rev. B 85, 174305 (2012).
[15] A. Cunsolo, Onset of a transverse dynamics in the THz spectrum of water, Mol. Phys. 111, 455 (2013).

[16] A. Cunsolo, C. N. Kodituwakku, F. Bencivenga, and A. H. Said, Shear propagation in the terahertz dynamics of water-glycerol mixtures, J. Chem. Phys. 139, 184507 (2013).

[17] S. Bellissima, S. De Panfilis, U. Bafile, A. Cunsolo, M. A. González, E. Guarini, and F. Formisano, The hydrogen-bond collective dynamics in liquid methanol, Sci. Rep. 6, 39533 (2016).

[18] T. Gaskell and S. Miller, Longitudinal modes, transverse modes and velocity correlations in liquids: I, J. Phys. C: Solid State Phys. 11, 3749 (1978).

[19] T. Gaskell and S. Miller, Longitudinal modes, transverse modes and velocity correlations in liquids: II, J. Phys. C: Solid State Phys. 11, 4839 (1978).

[20] T. Gaskell and S. Miller, Longitudinal modes, transverse modes and velocity correlations in liquids: III, J. Phys. C: Solid State Phys. 12, 2705 (1979).

[21] M. Canales and J. A. Padró, Dynamic properties of LennardJones fluids and liquid metals, Phys. Rev. E 60, 551 (1999).

[22] S. Kambayashi and Y. Hiwatari, Molecular-dynamics study of dynamical properties of dense soft-sphere fluids: The role of short-range repulsion of the intermolecular potential, Phys. Rev. E 49, 1251 (1994).

[23] S. Bellissima, M. Neumann, E. Guarini, U. Bafile, and F. Barocchi, Density of states and dynamical crossover in a dense fluid revealed by exponential mode analysis of the velocity autocorrelation function, Phys. Rev E 95, 012108 (2017).

[24] E. Guarini, S. Bellissima, U. Bafile, E. Farhi, A. De Francesco, F. Formisano, and F. Barocchi, Density of states from mode expansion of the self-dynamic structure factor of a liquid metal, Phys. Rev. E 95, 012141 (2017).

[25] U. Bafile, E. Guarini, and F. Barocchi, Collective acoustic modes as renormalized damped oscillators: Unified description of neutron and x-ray scattering data from classical fluids, Phys. Rev. E 73, 061203 (2006).

[26] A. De Francesco, E. Guarini, U. Bafile, F. Formisano, and L. Scaccia, Bayesian approach to the analysis of neutron Brillouin scattering data on liquid metals, Phys. Rev. E 94, 023305 (2016).

[27] P. Hohenberg and W. Kohn, Inhomogeneous electron gas, Phys. Rev. 136, B864 (1964); W. Kohn and L. J. Sham, Self-consistent equations including exchange and correlation effects, ibid. 140, A1133 (1965).

[28] Y. Waseda and M. Ohtani, Static structure of liquid noble and transition metals by x-ray diffraction, Phys. Status Solidi B 62, 535 (1974). 
[29] I. Kaban and W. Hoyer, Interplay between atomic and electronic structure in liquid noble-polyvalent metal systems, J. NonCryst. Solids 312-314, 41 (2002).

[30] M. C. Bellissent, P. Desre, R. Bellissent, and G. Tourand, Neutron diffraction studies of liquid silver and liquid Ag-Ge alloys, J. Phys. 37, 1437 (1976).

[31] W. R. Osório, D. R. Leiva, L. C. Peixoto, L. R. Garcia, and A. Garcia, Mechanical properties of $\mathrm{Sn}-\mathrm{Ag}$ lead-free solder alloys based on the dendritic array and $\mathrm{Ag}_{3} \mathrm{Sn}$ morphology, J. Alloys Compd. 562, 194 (2013).

[32] H. Nishikawa and N. Iwata, Formation and growth of intermetallic compound layers at the interface during laser soldering using $\mathrm{Sn}-\mathrm{Ag}-\mathrm{Cu}$ solder on a $\mathrm{Cu}$ pad, J. Mater. Process. Technol. 215, 6 (2015).

[33] A. A. El-Daly, A. M. El-Taher, and S. Gouda, Development of new multicomponent $\mathrm{Sn}-\mathrm{Ag}-\mathrm{Cu}-\mathrm{Bi}$ lead-free solders for lowcost commercial electronic assembly, J. Alloys Compd. 627, 268 (2015).

[34] B. G. del Rio, O. Rodriguez, L. E. González, and D. J. González, First principles determination of static, dynamic and electronic properties of liquid Ti near melting, Comput. Mater. Sci. 139, 243 (2017).

[35] M. Marqués, L. E. González, and D. J. González, Ab initio study of the structure and dynamics of bulk liquid Fe, Phys. Rev. B 92, 134203 (2015)

[36] B. G. del Rio, L. E. González, and D. J. González, Ab initio study of several static and dynamic properties of bulk liquid Ni near melting, J. Chem. Phys. 146, 034501 (2017).

[37] B. G. del Rio and L. E. González, Longitudinal, transverse, and single-particle dynamics in liquid $\mathrm{Zn}$ : $A b$ initio study and theoretical analysis, Phys. Rev. B 95, 224201 (2017).

[38] M. Marqués, L. E. González, and D. J. González, Pressureinduced changes in structural and dynamic properties of liquid Fe close to the melting line. An $a b$ initio study, J. Phys.: Condens. Matter 28, 075101 (2016).

[39] N. Jakse and T. Bryk, Pressure evolution of transverse collective excitations in liquid $\mathrm{Al}$ along the melting line, J. Chem. Phys. 151, 034506 (2019).

[40] T. Bryk, T. Demchuk, and N. Jakse, Atomistic structure and collective dynamics in liquid $\mathrm{Pb}$ along the melting line up to 70 GPa: A first-principles molecular dynamics study, Phys. Rev. B 99, 014201 (2019).

[41] F. Barocchi, U. Bafile, and M. Sampoli, Exact exponential function solution of the generalized Langevin equation for autocorrelation functions of many-body systems, Phys. Rev. E 85, 022102 (2012).

[42] F. Barocchi and U. Bafile, Expansion in Lorentzian functions of spectra of quantum autocorrelations, Phys. Rev. E 87, 062133 (2013).

[43] F. Barocchi, E. Guarini, and U. Bafile, Exponential series expansion for correlation functions of many-body systems, Phys. Rev. E 90, 032106 (2014).

[44] S. Bellissima, M. Neumann, E. Guarini, U. Bafile, and F. Barocchi, Time dependence of the velocity autocorrelation function of a fluid: An eigenmode analysis of dynamical processes, Phys. Rev. E 92, 042166 (2015).

[45] E. Guarini, M. Neumann, U. Bafile, S. Bellissima, and D. Colognesi, Dynamical Origin of the Total and Zero-Point Kinetic Energy in a Quantum Fluid, Phys. Rev. Lett. 123, 135301 (2019).
[46] E. Guarini, M. Neumann, S. Bellissima, D. Colognesi, and U. Bafile, Density dependence of the dynamical processes governing the velocity autocorrelation function of a quantum fluid, Phys. Rev. E 100, 062111 (2019).

[47] D. S. Sivia and W. David, A Bayesian approach to extracting structure-factor amplitudes from powder diffraction data, Acta Crystallogr. A 50, 703 (1994).

[48] D. S. Sivia and C. J. Carlile, Molecular spectroscopy and Bayesian spectral analysis-how many lines are there? J. Chem. Phys. 96, 170 (1992).

[49] D. S. Sivia, W. A. Hamilton, and G. S. Smith, Analysis of neutron reflectivity data: Maximum entropy, Bayesian spectral analysis and speckle holography, Phys. B 173, 121 (1991).

[50] A. De Francesco, L. Scaccia, R. B. Lennox, E. Guarini, U. Bafile, U. P. Falus, M. Maccarini, Model-free description of polymer-coated gold nanoparticle dynamics in aqueous solutions obtained by Bayesian analysis of neutron spin echo data, Phys. Rev. E 99, 052504 (2019).

[51] A. De Francesco, L. Scaccia, M. Maccarini, F. Formisano, E. Guarini, U. Bafile, and A. Cunsolo, Interpreting the terahertz spectrum of complex materials: The unique contribution of the Bayesian analysis, Materials 12, 2914 (2019).

[52] V. F. Sears, Neutron scattering lengths and cross sections, Neutron News 3, 26 (1992).

[53] CRC Handbook of Chemistry and Physics, 59th ed., edited by, R. C. Weast (CRC Press, Boca Raton, 1979).

[54] S. Blairs, Correlation between surface tension, density, and sound velocity of liquid metals, J. Colloid Interface Sci. 302, 312 (2006).

[55] D. Aisa et al., The development of the BRISP spectrometer at the Institut Laue-Langevin, Nucl. Instrum. Methods Phys. Res. A 544, 620 (2005).

[56] D. Aisa et al., BRISP: A new thermal-neutron spectrometer for small-angle studies of disordered matter, J. Non-Cryst. Solids 352, 5130 (2006).

[57] F. Formisano, A. De Francesco, E. Guarini, A. Laloni, A. Orecchini, C. Petrillo, W.-C. Pilgrim, D. Russo, and F. Sacchetti, The neutron spectrometer BRISP: A new approach to the study of excitations in condensed matter at low momentum transfer in the milli-eV energy region, J. Phys. Soc. Jpn. 82, SA028 (2013).

[58] See Supplemental Material at http://link.aps.org/supplemental/ 10.1103/PhysRevB.102.054210, which includes Refs. [59-63], for details on the BRISP software and on the multiple scattering evaluation. Example plots about the various contributions to multiple scattering are provided, as well as indications about the source codes we developed for this purpose.

[59] D. Richard, M. Ferrand, and G. J. Kearley, J. Neutron Res. 4, 33 (1996).

[60] Instructions for the use of LAMP can be found at https:/www.ill.eu/users/support-labs-infrastructure/softwarescientific-tools/lamp/.

[61] A. De Francesco, U. Bafile, F. Formisano, and E. Guarini, Efficient implementation of multiple scattering Monte Carlo estimates in time-of-flight neutron spectrometry exploiting widearea detectors, J. Phys.: Conf. Ser. 340, 012024 (2012).

[62] MATLAB, vers. 7.4 (R2007a) (The MathWorks, Natick, MA, 2007).

[63] E. Guarini, A. Orecchini, F. Formisano, F. Demmel, C. Petrillo, F. Sacchetti, U. Bafile, and F. Barocchi, Self-dynamics of 
hydrogen gas as probed by means of inelastic neutron scattering, J. Phys.: Condens. Matter 17, 7895 (2005).

[64] M. Sampoli, U. Bafile, E. Guarini, and F. Barocchi, Collective dynamics and molecular interactions in liquid $\mathrm{CO}_{2}$ by inelastic neutron scattering and computer simulations, Phys. Rev. B 79, 214203 (2009).

[65] P. Protopapas, H. C. Andersen, and N. A. D. Parlee, Theory of transport in liquid metals. I. Calculation of self-diffusion coefficients, J. Chem. Phys. 59, 15 (1973).

[66] U. Bafile, E. Guarini, M. Sampoli, and F. Barocchi, Characteristic times in the nanometer-picosecond translational collective dynamics of molecular liquids, Phys. Rev. E 80, 040201(R) (2009).

[67] P. Giannozzi et al., QUANTUM ESPRESSO: A modular and open-source software project for quantum simulations of materials, J. Phys.: Condens. Matter 21, 395502 (2009).

[68] J. P. Hansen and I. R. McDonald, Theory of Simple Liquids (Academic Press, London, 1986).
[69] M. P. Allen and D. J. Tildesley, Computer Simulation of Liquids (Clarendon Press, Oxford, 1987).

[70] I. M. de Schepper, P. Verkerk, A. A. van Well, and L. A. de Graaf, Short-Wavelength Sound Modes in Liquid Argon, Phys. Rev. Lett. 50, 974 (1983).

[71] T. Bryk, Non-hydrodynamic collective modes in liquid metals and alloys, Eur. Phys. J.: Spec. Top. 196, 65 (2011).

[72] T. Bryk and J.-F. Wax, A search for manifestation of two types of collective excitations in dynamic structure of a liquid metal: $A b$ initio study of collective excitations in liquid $\mathrm{Na}$, J. Chem. Phys. 144, 194501 (2016).

[73] S. Bellissima, M. A. González, U. Bafile, A. Cunsolo, F. Formisano, S. De Panfilis, and E. Guarini, Switching off hydrogen-bond-driven excitation modes in liquid methanol, Sci. Rep. 7, 10057 (2017).

[74] L. van Hove, The occurrence of singularities in the elastic frequency distribution of a crystal, Phys. Rev. 89, 1189 (1953). 